\title{
Modalités du don. Temporalité et tradition dans les mosaïques de Sainte-Sophie
}

Tania Kambourova

\section{(2) OpenEdition}

12 Journals

Édition électronique

URL : http://journals.openedition.org/imagesrevues/1161

DOI : 10.4000/imagesrevues. 1161

ISSN : $1778-3801$

Éditeur :

Centre d'Histoire et Théorie des Arts, Groupe d'Anthropologie Historique de l'Occident Médiéval, Laboratoire d'Anthropologie Sociale, UMR 8210 Anthropologie et Histoire des Mondes Antiques

\section{Référence électronique}

Tania Kambourova, "Modalités du don. Temporalité et tradition dans les mosaïques de SainteSophie », Images Re-vues [En ligne], Hors-série 1 | 2008, mis en ligne le 01 février 2008, consulté le 30 janvier 2021. URL : http://journals.openedition.org/imagesrevues/1161 ; DOI : https://doi.org/ 10.4000/imagesrevues. 1161

Ce document a été généré automatiquement le 30 janvier 2021.

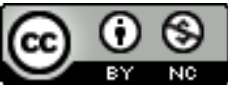

Images Re-vues est mise à disposition selon les termes de la Licence Creative Commons Attribution Pas d'Utilisation Commerciale 4.0 International. 


\title{
Modalités du don. Temporalité et tradition dans les mosaïques de Sainte-Sophie
}

\author{
Tania Kambourova
}

Nous aborderons la problématique de la temporalité et de la tradition des images à travers une troisième notion qui est celle du don. En effet, nous faisons appel à un autre concept pour accéder aux catégories qui nous intéressent, car l'interrogation sur le don nous permettra de poser indirectement des questions sur la temporalité et l'utilisation de la tradition

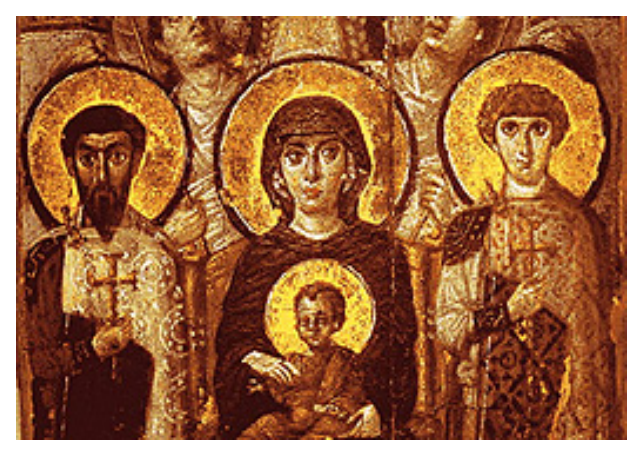
dans les images. Ainsi, les mosaïques byzantines de Sainte-Sophie (Constantinople, l'actuel Istanbul) seront examinées sous deux aspects: l'un se rapporte à l'image comme don à Dieu à une époque particulière, celle de la période post-iconoclaste qui se caractérise par l'invention d'une tradition des images saintes afin de justifier l'essor de l'iconographie nouvelle - et la mosaïque de la Théotokos dans l'abside est analysable dans ce sens; le second concerne l'image du don à Dieu qui, malgré ses variations, se situe dans une tradition prétendue immuable, refuse la temporalité et veut participer de l'eschatologie en faisant, en quelque sorte, comparaître le donateur devant le Juge suprême. A premier abord, l'image ne s'intéresse pas au temporel, mais à l'intemporel, et ce qui prime est le souci de présenter l'« innovation » comme une tradition. Les mosaïques des basileis offrent un exemple de cette pensée en images. 
Fig.1

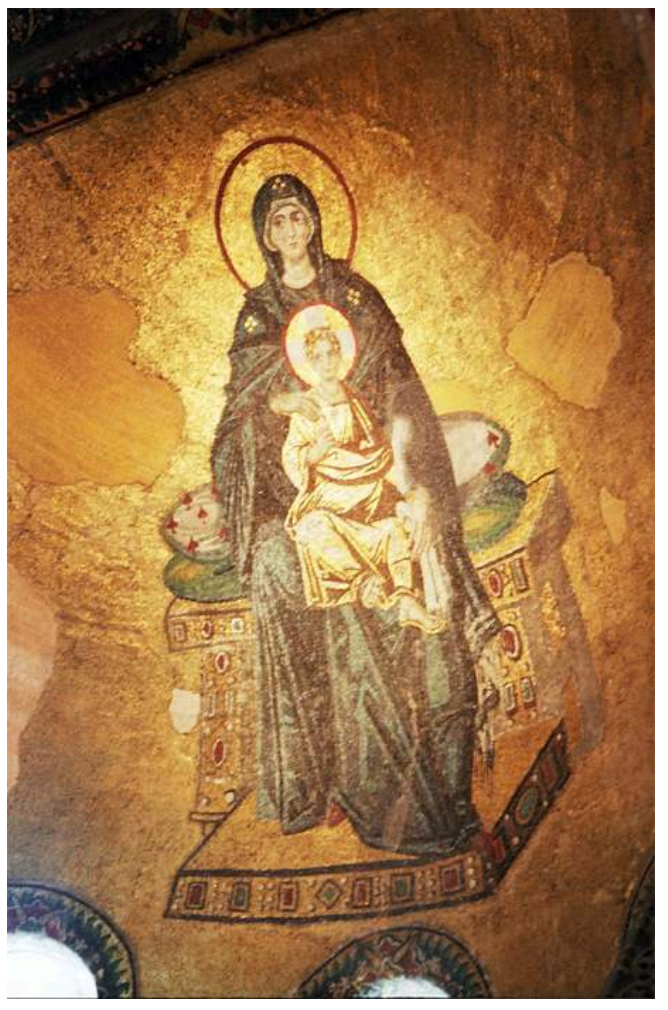

Theotokos. Conque de l'abside, Sainte-Sophie, IX s. ( ?), (détail sans l'archange) ; photographie : Claire Thomas

Fig.2

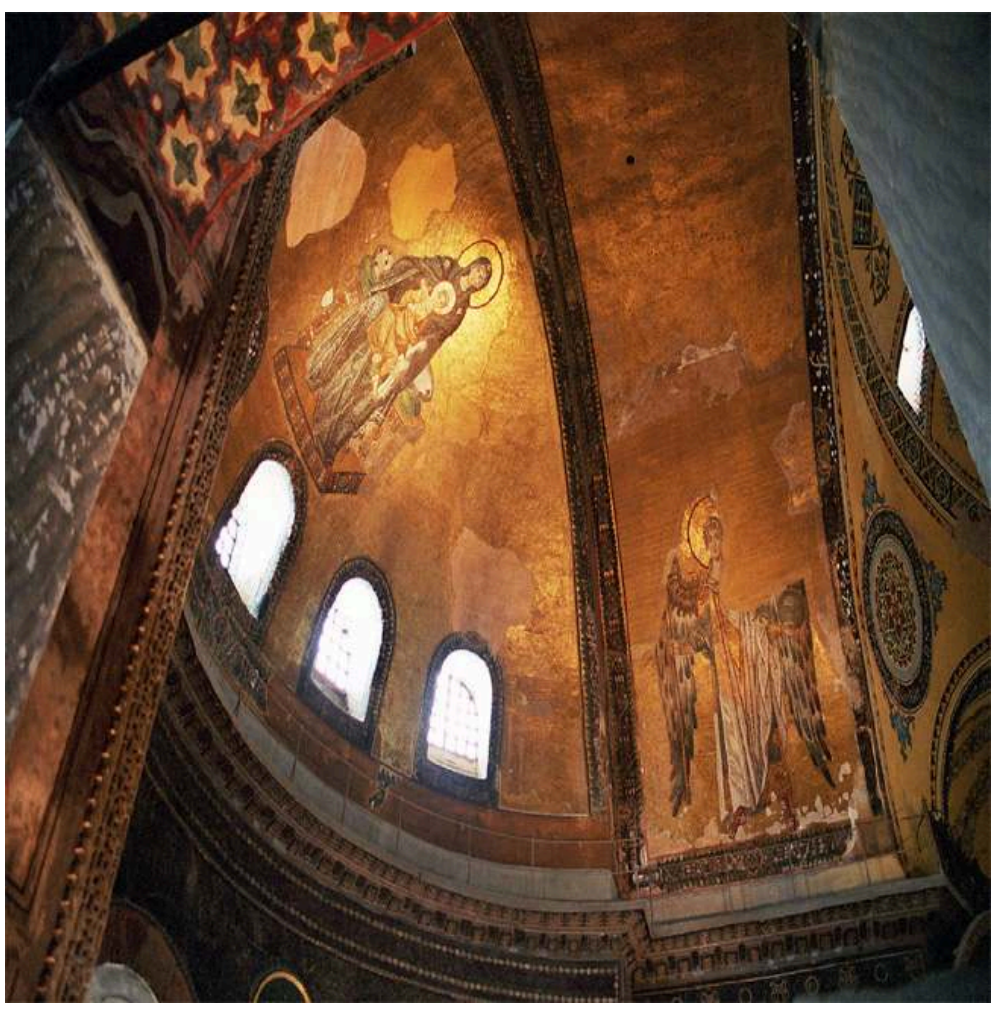

Theotokos et ange. Conque de l'abside, Sainte-Sophie, IXe s. ( ?). 
2 Les mosaïques conservées à Sainte-Sophie sont assez fragmentaires et, bien que très connues et étudiées, elles n'ont toujours pas livré leurs secrets. Parmi les plus remarquables à mentionner, il y a celles de la Théotokos à l'Enfant dans la conque de l'abside (fig. 1 et 2 ) et celles des panneaux des basileis (fig. 5-10). La question du commanditaire et du destinataire des mosaïques est liée à celle de la problématique du don, et, donc, pourra s'avérer lourde de conséquences pour notre analyse.

\section{L'image comme don à Dieu : le panneau absidal de la Théotokos}

3 La seconde victoire des images a lieu le 11 mars $843^{1}$. Pourtant, les images mettent du temps avant de commencer à orner les parois des églises. Ainsi, on suppose que la première mosaïque post-iconoclaste est née de l'intention du patriarche Photius (qui arrive au pouvoir en 857). Faisant partie d'un programme iconographique nouvellement codifié, la mosaïque de la Théotokos dans la conque de l'abside connaît une inauguration officielle en 867 , le jour de Pâques, en présence des deux empereurs Michel III et Basile Ier ${ }^{2}$. Nous allons revenir sur le sermon du patriarche. Mais dès à présent, il faudrait insister sur le soin de faire coïncider, comme il était souvent l'usage, lors des couronnements impériaux, par exemple, un événement particulièrement important du calendrier chrétien avec une actualité de l'histoire humaine et une activité liturgique. Plusieurs temps se confondent dans une temporalité complexe : le temps de la célébration liturgique, celui de la fête chrétienne et encore celui de la célébration inaugurale d'une image monumentale dans l'église, qui est de l'ordre de l'historique. Ainsi, le temps et l'histoire des humains s'inscrivent dans ceux du divin, sur lesquels ils sont réglés. Ce fonctionnement par coïncidence des temps humains et divins modèle l'événement en le sacralisant. Les actions humaines scandent et actualisent le divin en le faisant intervenir directement dans le registre terrestre. Les deux mondes, les deux temporalités se rencontrent. Mais ces actions humaines sont d'autant plus complexes à situer sur la courbe temporelle du présent ou du devenir qu'elles sont orientées vers une conformité avec le passé. Pour les Byzantins, il était extrêmement important de faire coïncider les événements du présent avec ceux du passé, et inversement même, se livrant à certaines interprétations que l'on pourrait qualifier aujourd'hui de "manipulations $»^{3}$. Par conséquent, on comprend mieux le poids de la tradition et le souci d'inscrire la nouveauté, l'innovation sous la garantie du passé.

4 La composition de la mosaïque de la Mère de Dieu est très simplifiée. Aucun cadre, mises à part les contraintes architecturales, ne vient limiter l'installation du divin en image. La Théotokos était à l'époque flanquée des deux archanges Gabriel (à droite) et Michel (à gauche, qui n'a pas subsisté) ${ }^{4}$. C'est la question de l'inscription dédicatoire qui va nous permettre de nous intéresser à l'inscription de cette image dans la tradition. Elle est la suivante : «Les images que les imposteurs ont détruites, les empereurs pieux les ont de nouveau relevées $»^{5}$.

5 L'inscription insiste sur l'existence d'images auparavant. Pourtant, aujourd'hui, les historiens et archéologues sont unanimes pour penser qu'à part plusieurs croix sur fond d'or et des motifs ornementaux datant de l'époque de Justinien, il n'y avait pas eu d'autre décoration dans l'église avant l'iconoclasme. Alors dans quel mécanisme de 
pensée s'inscrit l'insistance sur les images qui auraient été de nouveau relevées par les empereurs pieux?

6 En fait, l'inscription d'un phénomène nouveau dans une tradition ancienne et la transformation des événements du passé pour les faire correspondre avec ceux du présent n'ont rien pour nous étonner, car ce sont des moyens très byzantins pour « déguiser » les mini-ruptures en continuité.

\section{Fig.3}

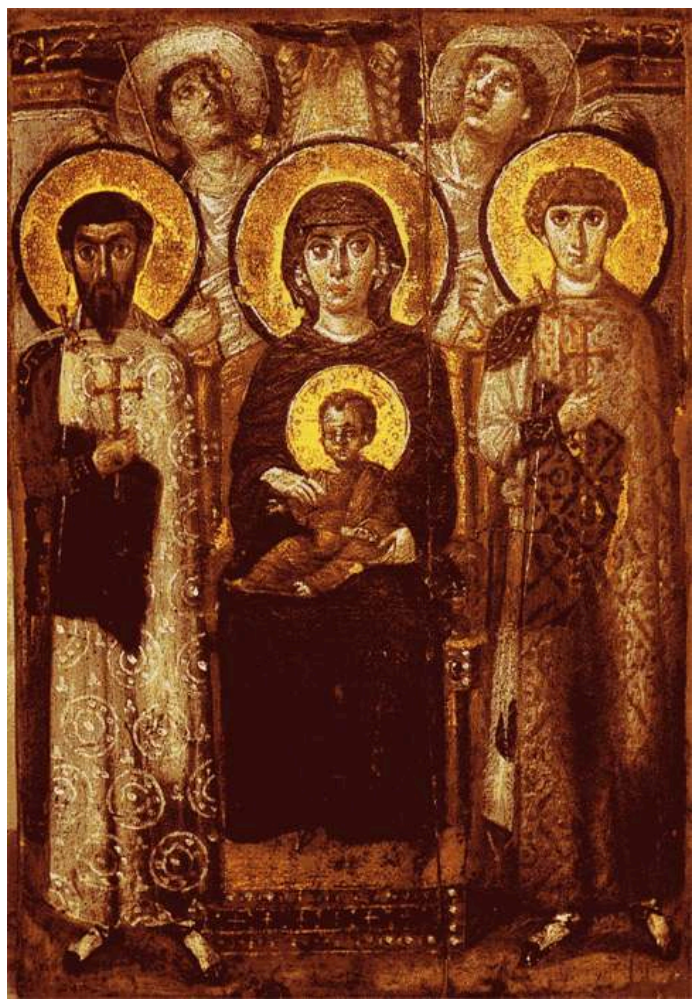

Theotokos, entre les saints Théodore et Georges. Vle s., 68 cm, Sinaï, Sainte-Catherine 
Fig. 4

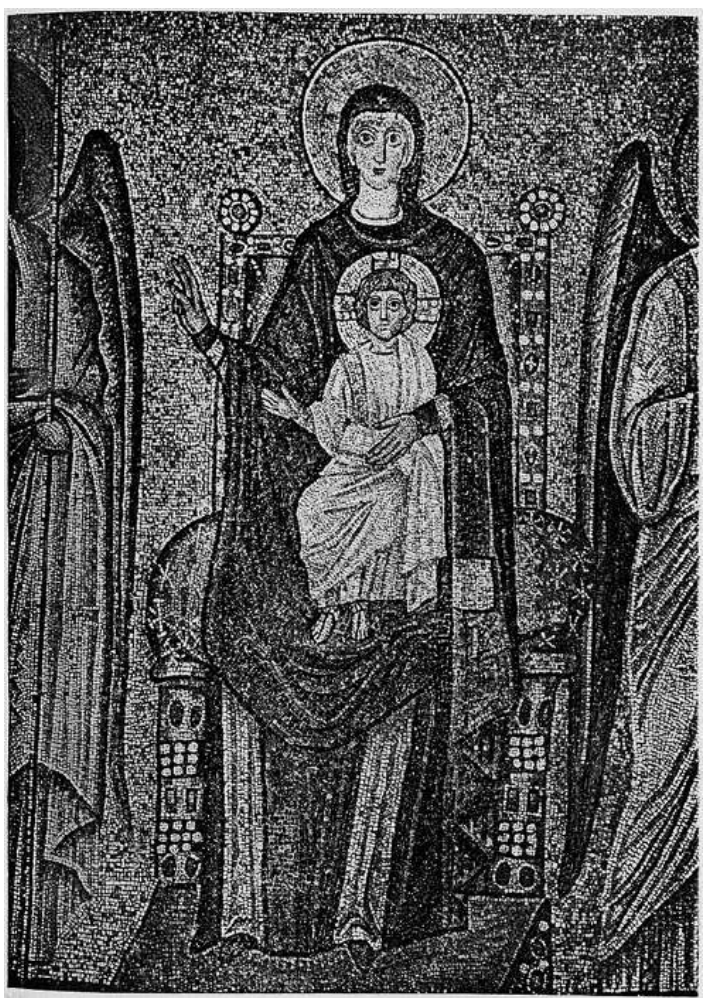

Vierge entourée d'anges, Ravenne, Saint-Apollinaire-le-Neuf,Vle s.

7 Ainsi, le schéma iconographique de la Vierge correspond en grande partie au modèle de représentation de la Théotokos avant l'époque iconoclaste, et qui était, sans doute, le plus répandu ${ }^{6}$. Pourtant, dans l'iconographie monumentale, un attribut peu utilisé est apparu - le mouchoir, la composition s'est simplifiée, a gagné en hiératisme, fixité, dignité ; la Vierge occupe la place centrale qu'elle partage avec le Christ Enfant. Le sens aussi s'est déplacé : de l'idée triomphale de la Vierge protectrice de la ville, il a évolué vers celui de la Vierge Mère, matérialisant en image le dogme de l'Incarnation, intercédant auprès du Christ Juge pour le gendre humain, dans les compositions de Déisis (et dans celles du Jugement dernier) . $^{8}$

D'ailleurs, l'institution du dogme au VIII ${ }^{\mathrm{e}}$ siècle, à Nicée, est présentée comme une restauration de l'iconodoulie, alors qu'on sait pertinemment qu'auparavant, les images ne jouaient pas de rôle officiel dans la théologie et que l'Eglise n'avait promulgué aucun édit sur la question, ni avait pris une position quelconque. D'ailleurs, comme l'avait relevé déjà André Grabar, la première fois que celle-ci a dû intervenir a été pour les interdire. Cette décision était due en partie à leur multiplication incontrôlée, alors qu'elles n'avaient pas de véritable statut reconnu par l'Eglise, et, en partie, à leur usage magique, rappelant fortement les pratiques païennes, proches de l'idolâtrie 9 . Paradoxalement, sans doute, c'est suite à l'iconoclasme et à la contestation des images que s'est développée une véritable théorie, sinon théologie, de l'image.

Plusieurs mécanismes se mettent en place grâce à la volonté d'inscription de nouveaux dogmes dans une tradition tout en utilisant des éléments existants. L'oralité est invoquée pour justifier une existence des images remontant aux origines du christianisme. Les ennemis des images sont diabolisés et menacés de vengeance. L'un de leurs plus grands défenseurs est Jean Damascène qui argumente la représentation du 
divin comme rendue possible par l'Incarnation. A partir du moment où Dieu s'est révélé à travers son Fils, il peut être représenté. Ainsi, il développe l'idée d'une correspondance innée entre le monde concret et celui de l'au-delà, d'un mystérieux lien intrinsèque entre l'image (l'icône) et le prototype. L'argumentation du théologien est sur plusieurs plans. Elle est historique, se référant aux écritures bibliques et évangéliques, théologique, se fondant sur la nature humaine du Christ Dieu, didactique, chargeant pour longtemps les images de la tâche d'enseigner aux analphabètes, et, finalement, légitimatrice, inscrivant l'absence de textes justificateurs des images dans la tradition orale ${ }^{10}$.

10 L'icône se veut être une théophanie et un moyen de faire parvenir la vénération au modèle par l'image, et elle est ancrée dans une tradition qui, à défaut d'être écrite, valorise l'oralité, reposant sur les Pères de l'Eglise, les apôtres, la révélation de Dieu qui s'est fait homme. De plus, la vénération de la croix, universellement reconnue dans l'empire, est amalgamée avec celle, contestée, des images. La première victoire des images contribue à l'écriture de la théorie qui est officialisée au Septième concile de Nicée, en $787^{11}$.

11 Un autre mécanisme pour affirmer la légitimité du culte des images passe par le biais des icônes achéiropoètes - "non faites de mains d'homme ", qui sont à rapprocher du culte des reliques, de l'enseigne victorieuse du combat, l'image étant érigée en signe de victoire à l'exemple du labarum ${ }^{12}$. Une liste intéressante est dressée des icônes miraculeuses dans la Lettre des Patriarches d'Alexandrie, d'Antioche et de Jérusalem à l'Empereur Théophile, en l'an 836, écrite quelques années avant la victoire définitive du dogme des images ${ }^{13}$. Ce catalogue ouvre aussi la voie aux images «faites de main d'homme » qui sont, en quelque sorte, contaminées par le dogme de la présence divine dans les icônes, où le Christ est l'icône du Dieu inconnaissable et toute icône de lui, de sa mère, des saints est une restitution du prototype, une ressemblance avec lui. Ainsi les images saintes, ou les icônes, auraient une fonction de transcendance, d'intermédiaire, d'initiation, d'imitation, et seraient comme des portes vers une visualisation de l'au-delà, une correspondance établie entre les réalités concrètes et la pensée, assurant la communication entre les deux mondes. Les deux types d'icônes sont mises sur le même plan, assurant la médiation du divin dans sa corporalité, ou encore celle de l'image-corps qui saigne, se venge, agit, ou encore celle qui est thaumaturge ${ }^{14}$. Parmi les douze icônes de la Lettre, on peut citer les achéiropoètes comme celle du Christ d'Edesse, deux de la Vierge à Lydda (en Palestine), et d'autres peintes par l'homme, comme celle dite de Luc, mais toutes sont du Christ et de la Vierge, à l'exception d'une, celle d'André.

12 Il ne s'agit, évidemment, pas de discuter la véracité des propos, ni même de définir le moment exact de l'apparition des icônes, mais de montrer le mécanisme d'argumentation qui arrive au bout de deux, trois siècles à faire imposer le culte des images officiellement et à faire aboutir une résistance due à leur contestation. La victoire des images est présentée comme une victoire à la fois sur les infidèles et sur les empereurs chrétiens "hérétiques", menacés de la vengeance des icônes. Les images sont thaumaturges comme les reliques, victorieuses comme le labarum, efficaces comme les effigies funéraires. Un message voilé est adressé aux iconoclastes à travers l'histoire de leur péripétie, à savoir que l'icône-corps souffre, mais finit par se venger quand elle est profanée. Elle devient le médium pour la vénération de Dieu et un don en son honneur. 
13 Bien que les iconodoules présentent l'interdiction des images comme accidentelle et exceptionnelle, les iconoclastes sont loin d'être des hérétiques rivaux du Christ. La représentation du divin n'allait pas de soi, et encore moins l'association du basileus et du Christ. D'ailleurs, Peter Brown, dans son livre la Société et le sacré, replace le débat entre les iconodoules et les iconoclastes dans son contexte historique. En effet, l'auteur montre clairement comment la manipulation des sources et de l'idée de tradition à Byzance, notamment par les iconodoules, a pu instituer l'idée d'une existence ininterrompue des icônes dans l'Eglise encore au temps apostolique. L'auteur souligne la tendance chez les historiens byzantins de présenter le mouvement iconoclaste généralement comme atypique et non byzantin et les basileis comme des souverains rivalisant avec le Christ pour la priorité sur les images. Or, et Peter Brown s'applique à le prouver, Léon III (726-730), par exemple, avait pris « ses décisions sur les conseils de sérieux évêques provinciaux, dans une tentative purement byzantine d'apaiser Dieu, qui avait montré clairement sa colère contre le peuple chrétien par les invasions arabes et par des éruptions volcaniques. ${ }^{15}$. Les iconoclastes n'étaient pas des hérétiques jaloux en concurrence avec le Christ pour la royauté terrestre. Ils étaient, comme les autres souverains chrétiens, des imitateurs et des serviteurs du Christ. Ils vénéraient, d'ailleurs, la croix. En effet, l'iconoclasme était issu d'un débat dans la société sur le sacré. Pour les souverains iconoclastes, la présence du sacré pouvait se manifester seulement dans l'eucharistie, l'édifice de l'Eglise et la croix - elle-même donnée comme signe directement de Dieu. Constantin et Hélène l'avaient déjà proclamée signe de victoire et relique à vénérer. Quant aux autres images, les «icônes ne pouvaient être sacrées parce qu'elles n'avaient reçu aucune consécration d'en haut. $»^{16}$ Seulement, les souverains iconodoules, deux siècles plus tard, trouveront une autre forme pour exprimer leur dévotion.

Ayant resitué le débat autour des images, nous pouvons revenir au discours inaugural du patriarche Photius. Nous voyons à présent toute l'ampleur de cet acte. Le patriarche y reprend les arguments iconodoules et résume les griefs retenus contre les iconoclastes. Il compare aux dons eucharistiques, dont il ne cite que le vin, le don de l'image pour honorer Dieu et insiste particulièrement sur l'Incarnation. La mosaïque est un digne présent impérial en contrepartie de la couronne reçue. Nous avons la chance d'avoir l'image et son interprétation de l'époque, et nous allons en reproduire quelques fragments : «...Aujourd'hui, nous avons deux empereurs bien-aimés et emplis de piété, le père et le fils, resplendissant tous deux de la pourpre souveraine. Leur orthodoxie est à l'origine de ce que nous pouvons voir maintenant, la représentation de la Vierge. Elle nous accueille, non par le don du vin, mais grâce à une belle image par laquelle la partie pensante de notre âme, nourrie par nos yeux et aidée dans son épanouissement vers l'amour divin de l'orthodoxie, parvient à la vision la plus claire de la vérité... Grâce à l'art, nous avons d'elle une représentation qui imite la réalité. Elle regarde l'enfant avec affection, pourtant elle a l'air détaché et lointain face à cet enfant surnaturel qui ne marque pas d'émotion. Si on lui demandait comment elle peut en même temps être vierge et mère, on a l'impression qu'elle pourrait répondre, tant la peinture fait paraitre réelles ses lèvres, closes et immobiles comme pour un sacrement ; on dirait la sérénité et la beauté de l'original... Contemplez la beauté et les ornements dont cette église avait été privée. Cela était dû à la main sacrilège des juifs, à un acte de haine; cette représentation est un don de l'amour divin, ainsi que le culte des saintes icônes... La doctrine orthodoxe a de nouveau éclairé le monde grâce aux commandements des empereurs et de Dieu, cette image est pour moi le commencement 
de l'orthodoxie, l'accomplissement de ce même règne tourné vers l'amour de Dieu... L'histoire des martyrs est contenue dans les livres, mais les peintures nous en offrent une vision bien plus vivante... De la même manière, dans le cas de la Vierge à l'Enfant, c'est en voyant plutôt qu'en entendant qu'on peut mieux comprendre le grand mystère de l'Incarnation. La mémoire est bien plus efficace lorsqu'elle agit par l'intermédiaire de la vue... Accorde à ceux que tu as faits empereurs sur la terre de consacrer le reste de l'église par de saintes images; comme ils sont les yeux de l'univers, protège-les comme la prunelle de tes yeux, préserve-les de tout mal, fais-les paraitre terribles à leurs ennemis et garde-les, ainsi que nous tous, dignes de ton royaume infini et sacré... $»^{17}$

Dans les dernières sections de son discours, Photius insiste sur la légitimité du pouvoir impérial et sur la piété des basileis, par opposition aux iconoclastes semi-barbares, en affirmant que leur position dans la société est un don de Dieu. Il offre à Dieu des prières pour les empereurs. Ses paroles suggèrent aussi que ces derniers étaient à l'origine des mosaïques de l'abside. Cela ne veut pas dire qu'il faille prendre ses paroles au pied de la lettre. Ce discours révèle la mentalité de l'époque et l'honneur rendu au souverain représentant de Dieu sur Terre et initiateur de tout acte de foi. Ainsi, les historiens ont pu voir dans les empereurs les commanditaires des mosaïques, alors qu'ils auraient pu en être les bienfaiteurs et/ou les destinataires ${ }^{18}$. Les mosaïques sont offertes en leur nom comme don aux forces célestes, en reconnaissance pour le don du pouvoir dont parle Photius. Les propos du patriarche sont très significatifs - par l'image, on rend grâce à Dieu.

16 Le discours du patriarche nous permet encore de voir comment on fait coïncider plusieurs temps : celui de l'Incarnation, du jour de Pâques (et de la Résurrection), de la fin de l'iconoclasme, de l'espoir que les actions des iconodoules soient reconnues et durent. La Vierge y est désormais dite intercédant pour le salut, enseignant la vénération, et son aspect victorieux est atténué au profit de celui de médium de l'Incarnation, de protectrice de l'humanité, en général, et des actions impériales, en particulier. Le discours installe la représentation dans la tradition, pour masquer des innovations décoratives et une nouvelle pensée figurative. "Contemplez la beauté et les ornements dont cette église avait été privée ", nous dit Photius, alors que l'on sait pertinemment qu'il n'y avait jamais eu de semblable mosaïque. D'ailleurs, si une décoration avait existé, il est plus probable qu'elle ait été semblable à celle des absides de Ravenne ou de Rome qui représentaient, sur un fond étoilé ou doré, soit le Christ, soit encore le saint de l'église en orant (et plus rarement la croix) ${ }^{19}$.

\section{L'image du don à Dieu : les panneaux des basileis}

17 A part d'être un don à Dieu, l'image peut aussi figurer un don à Dieu. Nous avons peu d'informations sur les commanditaires de ces mosaïques. Une inscription louait un empereur qui a refait la décoration après le tremblement de terre du 9 janvier 869 , mais son monogramme est détruit et nous ne savons pas quand exactement ont eu lieu ces travaux de restauration et concernaient-ils les panneaux des basileis ${ }^{20}$. Jusqu'à aujourd'hui sous les décorations ornementales ottomanes, seuls cinq panneaux impériaux ont été découverts dans l'église de Sainte-Sophie, transformée en mosquée après la prise de Constantinople le 29 mai $1453^{21}$. Un des plus intéressants et soulevant le plus grand nombre de questions est sans doute le panneau dit «de Léon VI » du narthex (fig. 5). L'image est absolument novatrice par le lien qu'elle crée entre 
l'empereur est le Christ. L'association du basileus et du Christ n'est pas courante. Avant l'iconoclasme, dans un autre contexte, et sur d'autres supports, André Grabar recense trois cas d'association entre $\mathrm{eux}^{22}$, mais il faudrait insister davantage sur l'absence de tout contact entre le souverain terrestre et le céleste. Avec l'établissement du culte des images, l'association de l'empereur et du Christ n'est plus seulement une juxtaposition, mais un lien. Ainsi, la présence du souverain est plus qu'un témoignage de piété et d'humilité, une audace. Jusque là, nulle relation entre eux n'avait osé se faire jour dans l'iconographie monumentale. Pourtant, comme pour reprendre des éléments antérieurs, l'empereur est représenté nimbé et sans nom. Tel était le cas des panneaux de Saint-Vital à Ravenne où, couronnés et nimbés, Justinien et Théodora offraient leurs dons, sans qu'une inscription ne les mentionne (fig. 13 et 14). A Sainte-Sophie déjà les premiers panneaux post-iconoclastes de saints portaient des inscriptions, similaires à celles présentant les empereurs modèles Constantin et Justinien - disposées à la verticale, le long du corps du personnage (fig. 6). Alors pourquoi ce choix d'anonymat, dans un panneau, considéré postérieur aux " portraits » de saints conservés à SainteSophie?

Fig.5

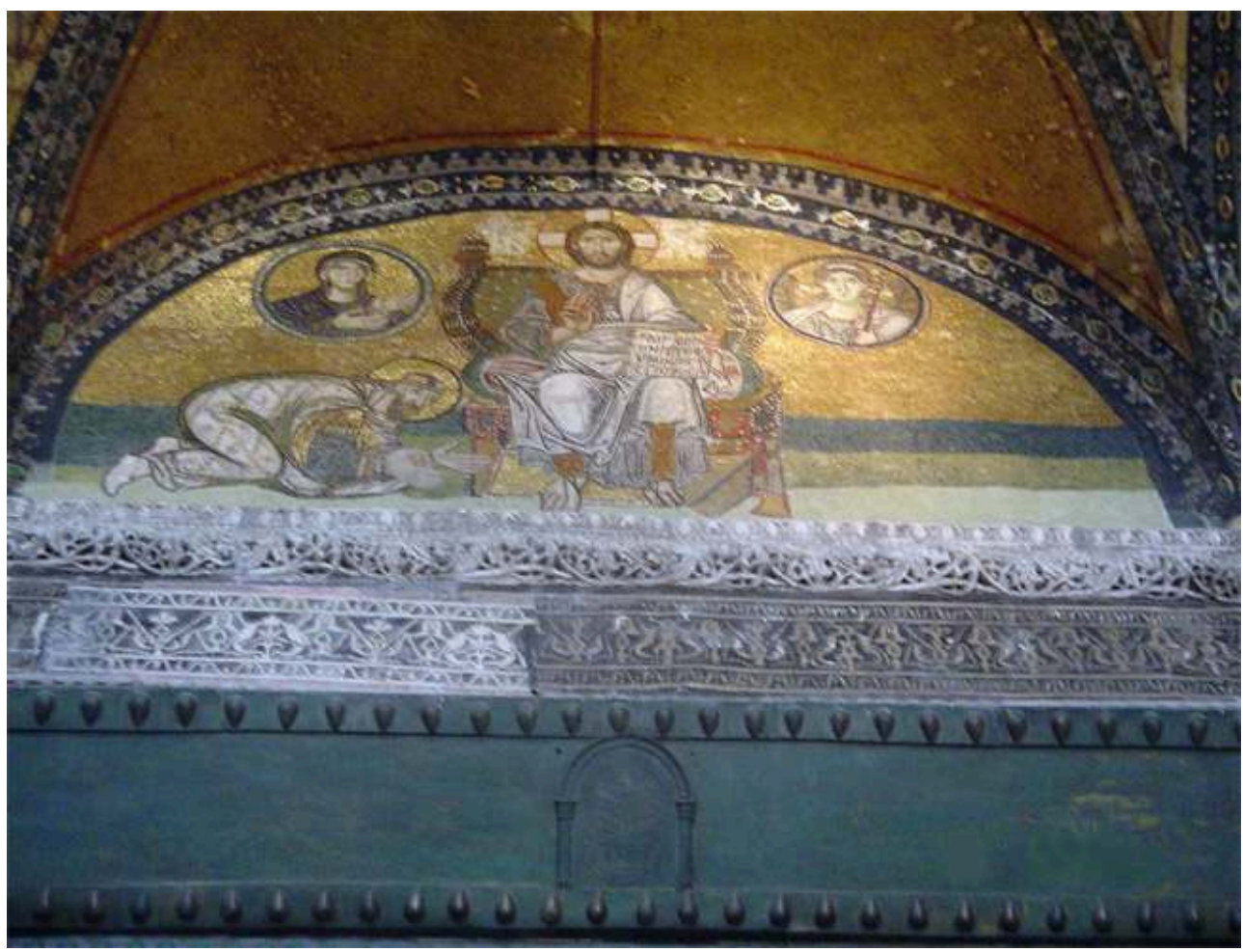

Basileus anonyme en proskynèse devant le Christ Lunette du narthex, Sainte-Sophie.

photographie : Svetlana Dimitrova 
Fig.13

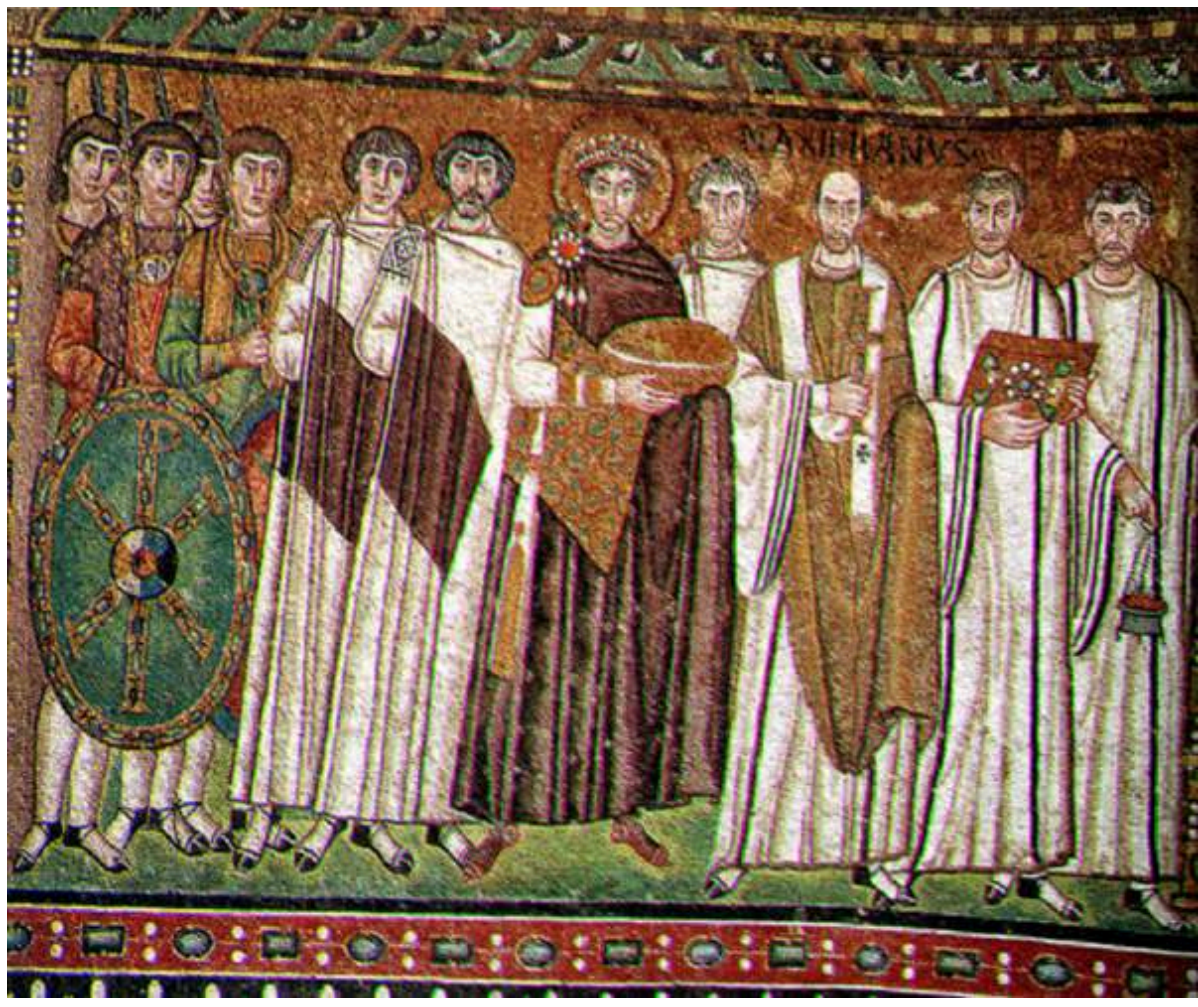

Justinien et sa cour. Presbyterium, nord, Saint-Vital, Ravenne, VIe s.

Fig. 14

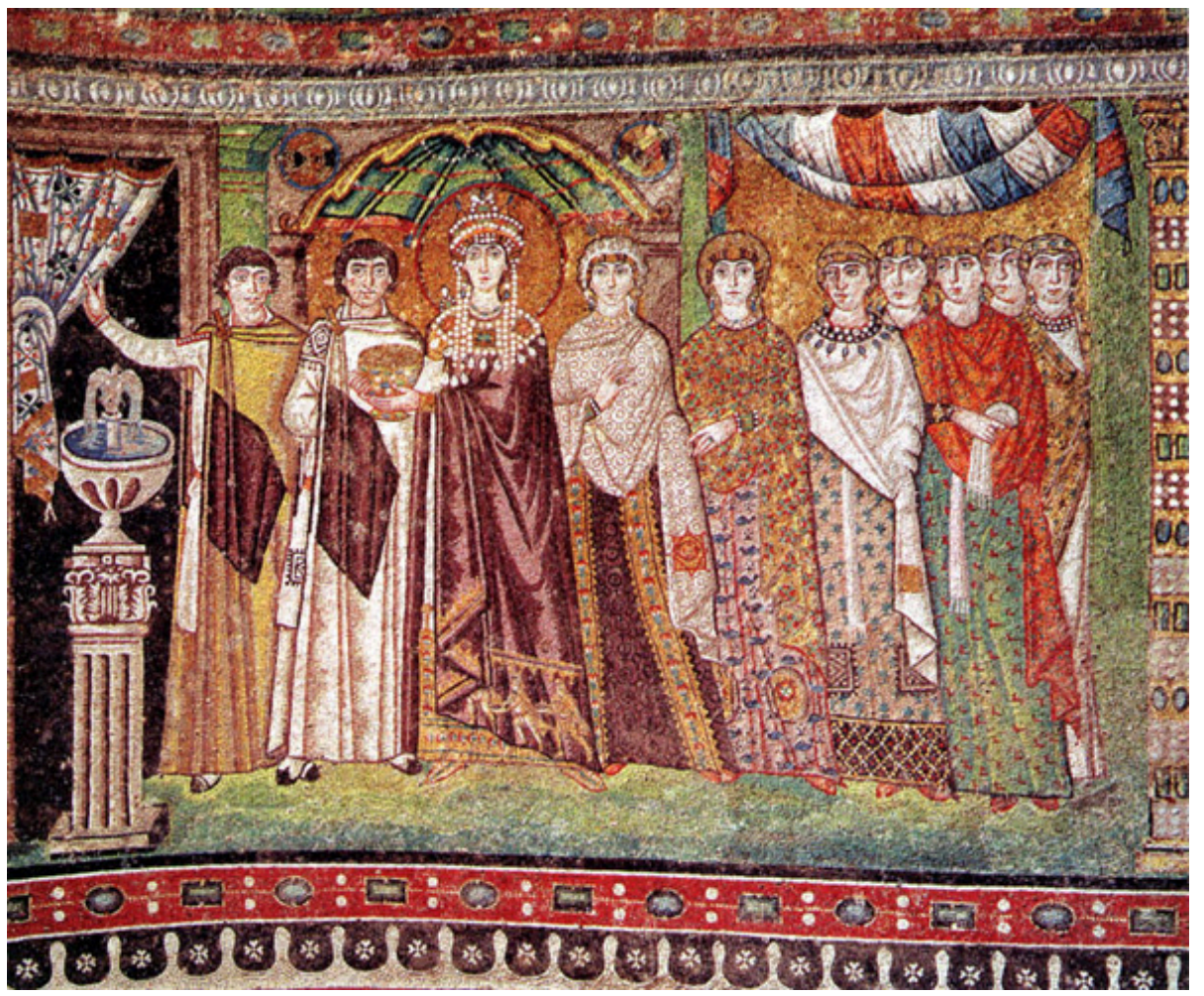

Théodora et sa cour. Presbyterium, sud, Saint-Vital, VIe s. 
Fig.6

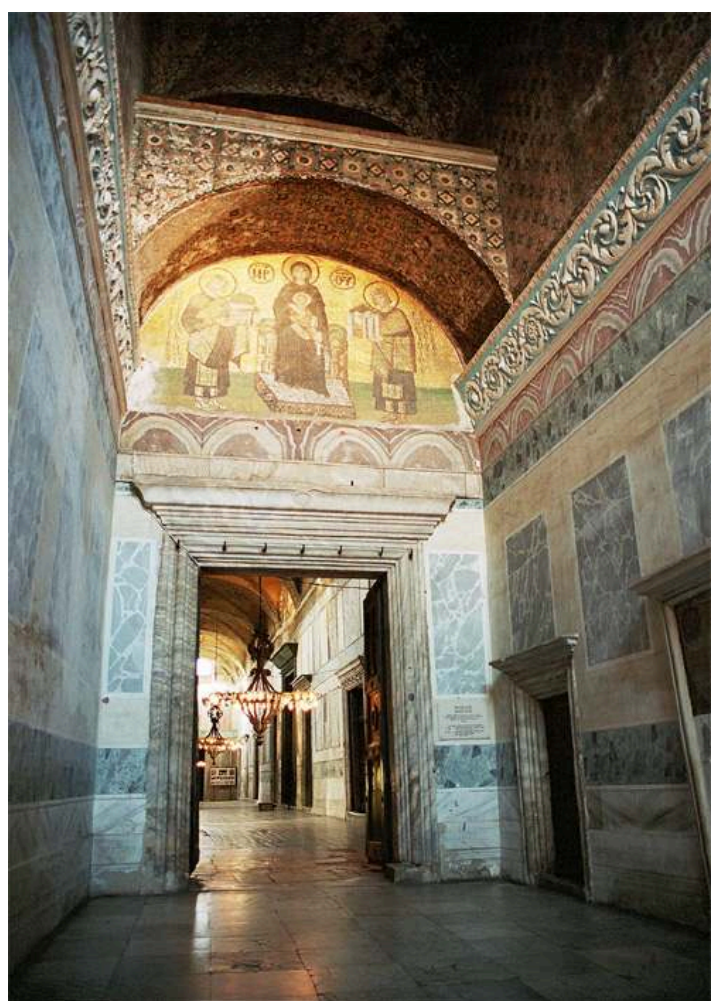

Constantin le Grand et Justinien présentant leurs dons à la Theotokos. Lunette du vestibule de SainteSophie.

photographie : Claire Thomas.

L'une des explications les plus courantes pour ce panneau est que l'empereur, identifié comme Léon VI, ait voulu se faire pardonner ses péchés, et notamment son quatrième mariage pour lequel il avait été interdit d'accès à l'église, par le patriarche Nicolas $I^{\text {er }}$ le Mystique. Le jour de l'Epiphanie de 907, il se serait prosterné devant lui pour demander le pardon de ses péchés. La mosaïque serait une évocation de cet événement, exécutée avant sa mort en 912. Pourtant, comment imaginer que la première mosaïque monumentale après la crise iconoclaste serait de caractère si personnel et « illustratif ", pour ne pas dire mimétique. Et quand bien même elle pourrait l'être, pourquoi resterait-il, à ce moment là, anonyme ? Simplement pour s'inscrire dans une tradition, alors que tous les autres personnages acteurs portent des inscriptions à Sainte-Sophie ? Nous avons fait cette précision de personnages acteurs pour l'opposer à celle de personnages montrés comme des icônes. Là encore, un motif iconographique de l'époque pré-iconoclaste, la tradition d'inscrire les saints dans un clipeus, fortement influencée par l'art funéraire romain de l'Antiquité, intervient dans l'usage actuel, mais modifiée ${ }^{3}$. Alors qu'auparavant seuls les visages étaient visibles, les mains des personnages apparaissent: il s'agit ici de la Vierge et d'un ange. D'ailleurs, c'est précisément la gestuelle qui pourrait permettre de lever une ambiguïté dans l'interprétation de la scène. Depuis la découverte de cette mosaïque se pose la question de la signification de la scène. Déjà Thomas Whittemore suggère l'ambiguïté entre une Déisis et une Annonciation (à cause de l'identification de l'archange comme Gabriel) ${ }^{24}$. Il rappelle, sans s'y arrêter, le rôle protecteur de la Vierge et de Gabriel figurant déjà dans 
le sceptre en ivoire de Berlin avec un empereur identifié comme Léon $\mathrm{VI}^{25}$. C'est d'ailleurs les écrits de Léon VI sur l'Annonciation, qui ont fait privilégier l'identification de l'empereur aux pieds du Christ comme précisément Léon $\mathrm{VI}^{26}$. Or, il faut remarquer que Marie est souvent accompagnée d'Archange(s) dans l'iconographie du $\mathrm{VI}^{\mathrm{e}}$ siècle. A Sainte-Sophie aussi, entourée d'eux, elle est "sous leur garde" dans l'abside ${ }^{27}$. Les archanges peuvent être remarqués aussi dans des séquences narratives. Ainsi, un cas intéressant est représenté dans la scène narrative des femmes au tombeau, à SaintApollinaire-le-Neuf de Ravenne. Les personnages féminins font un geste vers le sépulcre vide du Christ, tout en regardant l'Archange qui leur répond par un geste de bénédiction, un regard et un sourire. S'il s'agissait à Sainte-Sophie d'une Annonciation, on pourrait imaginer un comportement plus impliqué de l'Archange qui pourrait faire un geste vers la Vierge, alors qu'il reste impassible, regardant devant lui, à la manière d'un garde ${ }^{28}$. La Vierge, par contre, dirige ses mains vers le Christ, dans un double geste de prière et de monstration, parallèle à celui de l'empereur. A notre avis, cette scène pourrait plutôt représenter une des premières occurrences d'une Déisis avec un Christ Juge trônant après l'ouverture du Livre ${ }^{29}$, un Archange témoin et garde, et la Vierge intercédant pour l'humanité, en général, et pour l'empereur, en particulier ${ }^{30}$. D'ailleurs le trône du Christ est un élément emprunté à l'iconographie antérieure et inclus, ici, dans cette nouvelle composition. Nous trouvons un Christ Juge assis sur le même somptueux trône dans une mosaïque à Ravenne, datant du VI ${ }^{\mathrm{e}}$ siècle ${ }^{31}$. Il s'agit d'une mosaïque du Christ Juge, entouré de chaque côté par un (arch)ange. Les anges regardent droit devant eux. En fait, comme nous l'avions vu dans le cas de la transformation de l'image triomphale de la Vierge et l'invocation de sa protection, entre le $\mathrm{VI}^{\mathrm{e}}$ et le $\mathrm{IX}^{\mathrm{e}}$ siècles, de la même manière, il a pu se produire un glissement de sens entre ce type de mosaïques du Christ Juge (époque pré-iconoclaste) et la variante plus clémente de l'intercession, à une époque plus tardive (post-iconoclaste). Ainsi, la composition avec la Vierge remplaçant l'ange a pu naître. Cette idée d'intercession est renforcée par le parallélisme des gestes de la Vierge et de l'empereur. S'il faut réellement identifier l'archange, ce serait, très probablement, celui qui avait annoncé la Bonne Nouvelle et qui témoigne, à présent, de la réalisation de la prophétie. Ainsi, nous pencherions pour l'interprétation d'une Déisis : une Déisis « en devenir » ou une Déisis au sens propre du mot (et non iconographique) ${ }^{32}$. En effet, il s'agit d'une prière de salut, sans que celui-ci ne soit nécessairement promis à l'empereur. Une fois de plus, nous voyons comment d'anciens éléments sont intégrés dans une iconographie novatrice par le sens et par la forme, mais qui, idéellement, se veut fidèle à la tradition ${ }^{33}$. D'ailleurs, l'iconographie se cherche, et les personnages restent, en partie, emprisonnés dans les anciennes formes de représentation, les empruntant exprès dans un souci de s'inscrire dans l'imagerie du passé pour justifier les éléments nouveaux comme traditionnels ${ }^{34}$. La raison de leurs productions reste cependant à être interprétée. Déjà Nikos Oikonomides suggérait que les mosaïques avaient pu être faites non sur l'ordre de l'empereur luimême mais présentées comme dons aux empereurs par leurs sujets ou par certains ecclésiastiques reconnaissants ${ }^{35}$. Mais même si elles avaient été faites d'après la demande de l'empereur, elles restent, par leur emplacement, destinées d'abord à lui et à Dieu, et non spécialement produites dans un but de «propagande ». 
Fig. 11

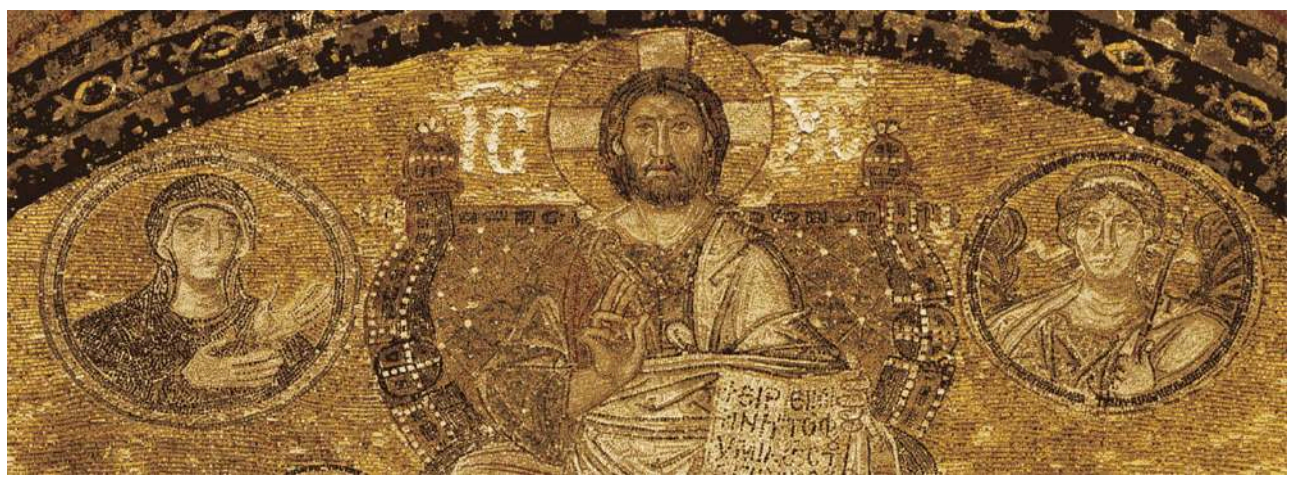

Détail de la mosaïque de Sainte-Sophie, narthex.

Fig. 12

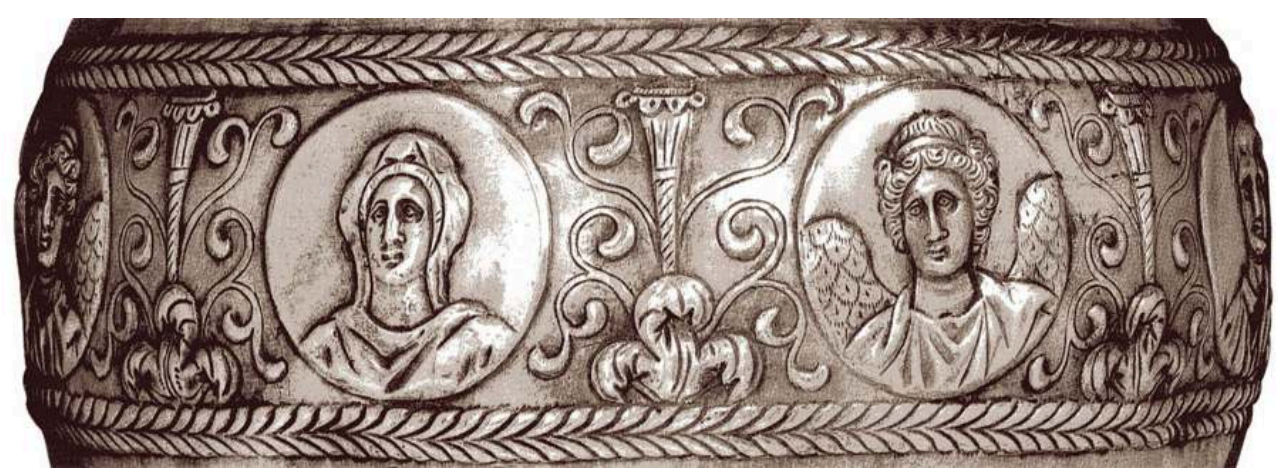

Détail du vase d'Emèse avec d'un côté le Christ entre deux apôtres et, de l'autre, la Vierge entre deux archanges. Vle s., argent, $\mathrm{H}: 45 \mathrm{~cm}$, Louvre. pourrait aussi que cette mosaïque se veuille exemplaire pour servir de modèle à imiter par les autres empereurs. La visée d'universalité pourrait ainsi évacuer l'individualité de la présence concrète. L'empereur aurait pu, donc, être laissé sans nom pour servir de modèle universel à la piété impériale et à l'expression de la vénération de Dieu. L'attitude de prière est loin de signifier une attitude plus humble après la « parenthèse » iconoclaste avec un abandon du trône au profit du Christ, mais bien au contraire, il s'agit d'une image très audacieuse ${ }^{36}$.

21 Non seulement l'identité est évacuée ici, mais aussi le temps. De la même manière que le don surnaturel de la couronne, par la Vierge, par un ange ou par le Christ lui-même, commençait, à Byzance, à être représenté en image pour attester la délégation du pouvoir par le Christ, la comparution des derniers temps, ou simplement la fin de la vie terrestre, aurait pu être imaginée et pensée en images par la représentation de l'empereur élu devant le Christ Juge ${ }^{37}$. Ainsi, l'empereur, en acteur de l'histoire sainte, court-circuite le temps des hommes pour se placer dans un temps eschatologique ${ }^{38}$. L'image situe le lieu de cette rencontre à la fois sur terre et au ciel par un dégradé de couleurs horizontal, aux pieds du Christ, et un fond doré sur lequel est inscrit le siège divin et la Déisis. L'empereur en prière partage aussi ces deux lieux ${ }^{39}$. Par l'image, l'empereur rend hommage à celui qui apporte le salut à l'homme. Son attitude pieuse « reproduit » celle que les sujets prennent pour témoigner leur hommage au souverain lors d'un cérémonial, le basileus, à son tour, se prosterne, mais en image, pour 
témoigner sa soumission au Roi des rois et l'assurer de sa foi. La prière de l'empereur suggère, ainsi, une portée eschatologique et sotériologique de l'image.

D'ailleurs, l'image devient désormais le lieu autorisé de cet échange et l'échange devient possible en image. L'intentionnalité d'exemplarité de la piété réussit à créer des émules. Par la suite, d'autres mosaïques expriment la piété envers le Christ (ou la Théotokos) et l'honneur rendu à l'empereur, de façon plus ou moins complexe, et sans qu'il s'agisse nécessairement de la représentation d'un empereur régnant. Une mosaïque de don à la Mère de Dieu est inscrite dans la lunette du vestibule sud (fig. 6). Deux empereurs se tiennent de part et d'autre de la Vierge, l'un étant Constantin et l'autre Justinien. Comme la mosaïque leur est postérieure, il est clair que c'est quelqu'un d'autre qui leur rend hommage (un autre empereur, le patriarche ou les deux).

Cette fois, c'est à travers la présence des personnages que la tradition est invoquée comme garantie. La nouvelle doctrine des images est appliquée à des anciens empereurs, sans qu'elle ait été pensée par eux. En effet, d'autres bienfaiteurs se cachent derrière la commande de ce panneau et expriment à travers lui leur reconnaissance et leur estime. La Théotokos, celle qui a donné naissance au Christ, reçoit d'un côté le don de la ville, offert par son fondateur, et celui de l'église, offert par celui qui l'a relevée après sa destruction. Le type de sa représentation a pu être rapproché de celui de la Vierge à l'Enfant recevant l'Adoration des Mages ${ }^{40}$. Plusieurs temporalités s'y rejoignent. Par la figuration, l'image renvoie à la souveraineté archétypale, biblique, christique, aux dons des Mages et à leur signification, mais par l'actualisation de ce modèle, deux temporalités humaines sont affichées comme modèle concomitant, celui du premier empereur chrétien et celui d'un empereur, qui à partir du IX ${ }^{\mathrm{e}}$ siècle, est essentiellement considéré comme le modèle du triomphateur ${ }^{41}$. Ce n'est pas le présent qui est rendu conforme au passé, c'est le passé qui est adapté au présent par l'utilisation de la tradition (et de ses illustres personnages) comme garantie. Les changements sont, en effet, acceptables que dans la limite où ils s'inscrivent dans la tradition. Chaque image qui rend hommage aux forces surnaturelles fait preuve d'une incroyable inventivité, cautionnée cependant par l'invariant traditionnel ou plutôt par la tradition invariante. Ainsi, un contemporain fait placer deux souverains du passé, vus à la lumière des attentes présentes, pour leur rendre hommage, mais aussi pour qu'ils servent d'exemple et pour que le souverain s'identifie à eux ${ }^{42}$. Ces souverains du passé sont offerts à l'imitation des contemporains grâce à l'image, mais ils sont actualisés en fonction de l'iconographie actuelle. Ils adoptent, en effet, les gestes et l'attitude, voire même le contexte qui les fait placer aux côtés de la Théotokos, en fonction de cette iconographie ${ }^{43}$. Les souverains anciens sont là à la fois pour servir de modèle aux actions d'évergétisme des contemporains et pour introduire une nouvelle attitude en image cautionnée par la garantie de leur exemplarité.

Par ailleurs, le modèle du don renvoie à un imaginaire qui a déjà pris forme dans le passé. Sans côtoyer, et encore moins faire un don directement à la Vierge ou au Christ (en image), Justinien et Théodora avaient été représentés dans un élan d'évergétisme au $\mathrm{VI}^{\mathrm{e}}$ siècle. Leur don portait la marque d'une référence explicite aux Mages, représentés sur le pan inférieur du manteau de Théodora ${ }^{44}$. La mosaïque de SainteSophie opère une actualisation de ce don par un autre don, celui de l'église. Cette fois, c'est le type iconographique de la Vierge trônant avec l'Enfant qui crée le parallélisme avec les Mages. La référence figurative aux Mages renvoie, à travers les offrandes, à la 
fois à l'annonce de la royauté du Christ et à son sacrifice, à son don de soi. D'ailleurs, la Mère de Dieu tient, dans la mosaïque de Sainte-Sophie, à la fois l'Enfant, le Dieu incarné et le mouchoir, signe de deuil, annonçant son sacrifice.

La représentation du don de Constantin reprend la forme du don d'objet ou d'église, mais innove entièrement dans la conception de l'objet. Son don est exceptionnel. Par son symbolisme unique, il témoigne de la force de la foi, de l'obligation de donner en retour. La fondation de la ville est pensée non seulement en terme d'hommage à celui qui lui a donné le pouvoir et la victoire, mais aussi à celle qui est la protectrice de la ville et qui intercède pour le pardon des péchés - la Vierge. C'est précisément cette complexité du don en retour qui servira de modèle iconographique à l'offrande impériale. Pour leur sacrifice, la Vierge, ou ailleurs le Christ trônant, reçoivent le don en retour, toutes temporalités confondues, dans une composition qui vise à inscrire l'innovation dans la tradition.

Deux autres mosaïques de la tribune sud évoquent des dons qui pourraient coïncider avec un moment précis de la pratique liturgique (fig. 8-10). Le schéma du panneau du vestibule est repris et il crée à son tour une tradition qui sert aussi d'exemple du bon comportement aux souverains suivants. Cette fois, c'est le Christ qui trône le livre fermé dans le panneau de Constantin et Zoé (fig. 9) et la Vierge à l'Enfant dans celui des Comnènes, calqué sur ce dernier (fig. 10) ${ }^{45}$. Les personnages de l'histoire contemporaine entourent, comme dans une Déisis, celui qui est prié de recevoir les dons et reprennent la disposition du panneau que nous venons de voir. Contrairement à ce que l'on peut croire, le souci de la mémoire n'intervient pas en premier lieu. Plus fort est celui de l'hommage, du désir de donner des modèles dignes d'être imités. Le fait que l'on représente un empereur sans lui donner de nom, alors que l'on institue parallèlement deux souverains comme les archétypes de la souveraineté chrétienne, le prouve de façon assez explicite. A cette explication se rattache aussi le fait que l'on ait effacé les noms des premiers bienfaiteurs sur le panneau dit de Zoé ${ }^{46}$. Par rapport aux panneaux précédents, les dons ont changé et correspondent, en partie, à une pratique liturgique concrète $^{47}$. Ce n'est évidemment pas une image mimétique du don, mais la reprise d'un élément du cérémoniel qui évoque le don dans l'image. Une fois de plus, les temporalités se confondent et l'élément concret est sorti de son contexte pour être inscrit dans une temporalité universelle, celle de l'image qui est aussi celle du temps où le Christ Juge viendra reconnaître les siens. 
Fig.8

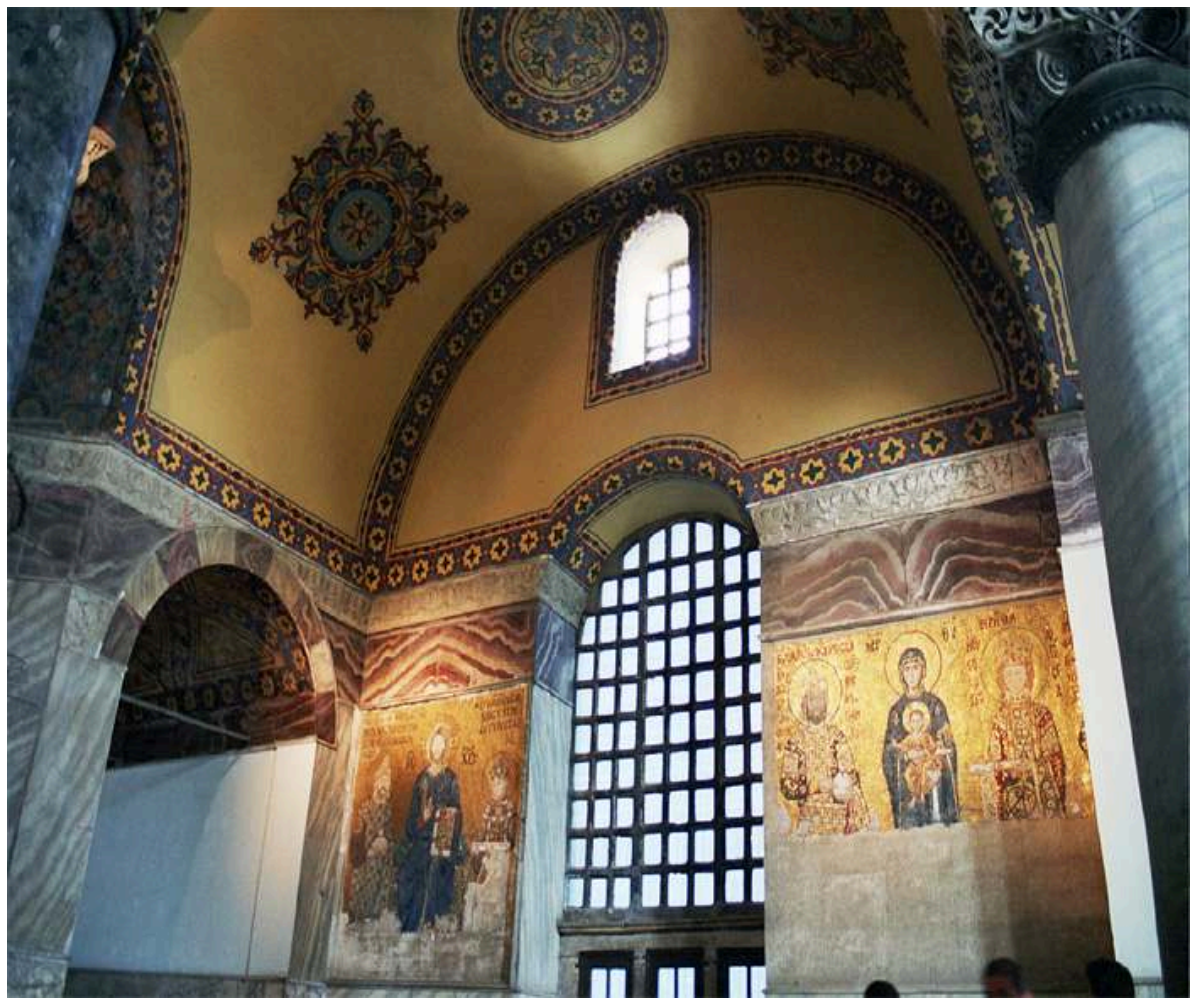

Vue d'ensemble des panneaux de la galerie sud, Constantin et Zoé, Jean II Comnène et Irène SainteSophie .

photographie : Claire Thomas.

Fig. 9

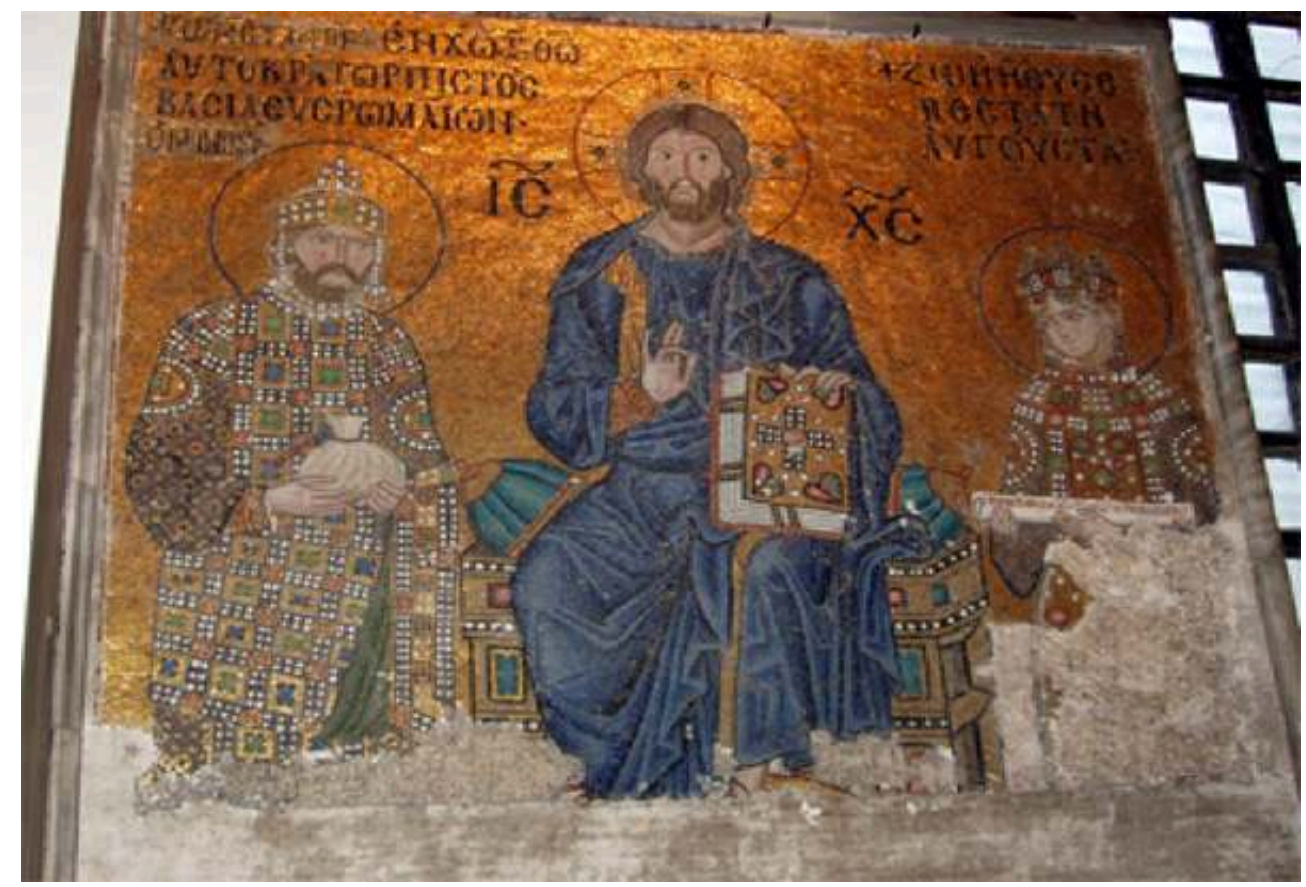

Détail du panneau retouché du Christ, entouré de Constantin IX et Zoé. Galerie sud, Sainte-Sophie, Xle s. (?).

photographie : Julian Dinkov. 
Fig. 10

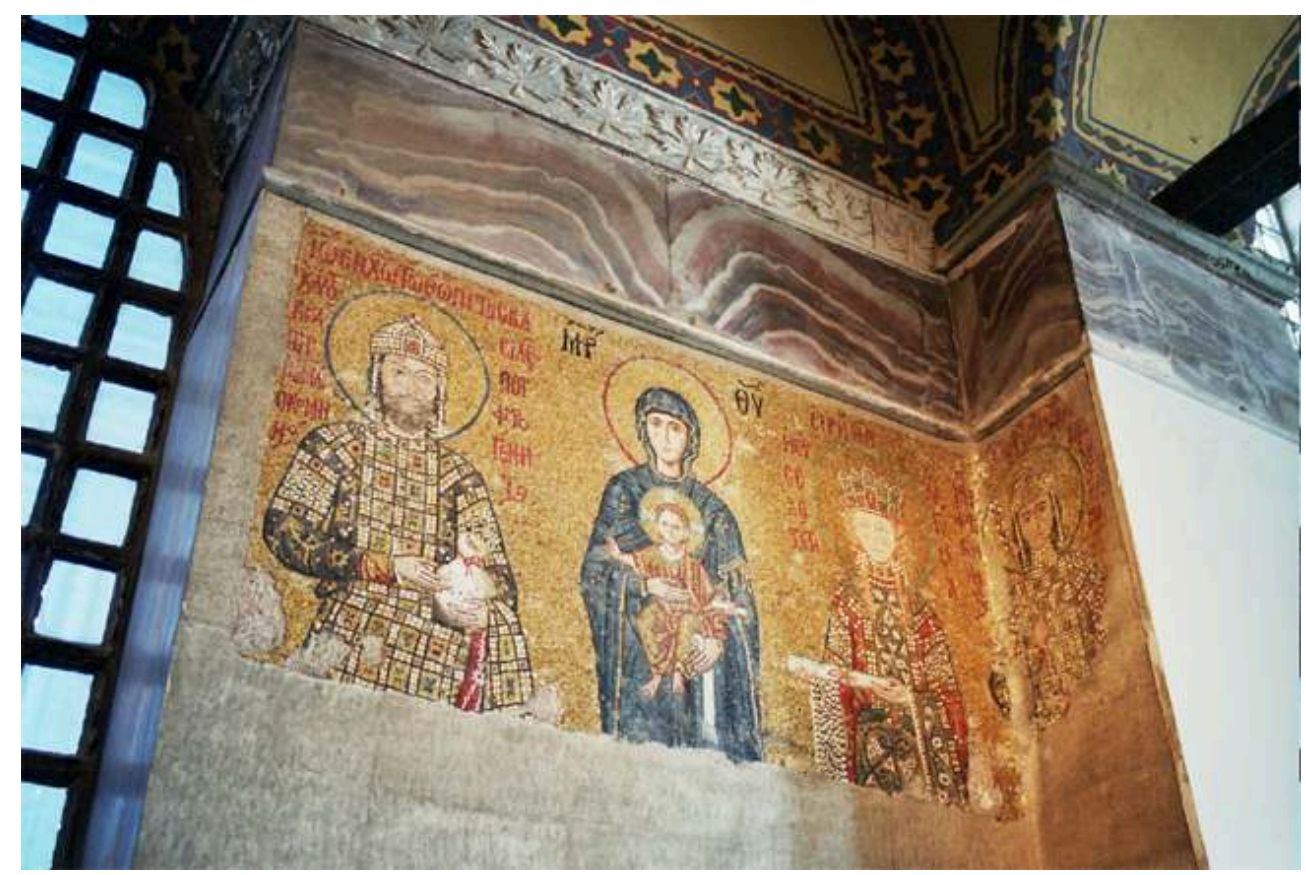

Détail du panneau des Comnènes (1118), mettant en image Jean II Comnène et Irène avec sur le pilastre jouxtant leur panneau celui de leur fils Alexis

Photographie : Claire Thomas

Dans la dernière image que nous évoquerons, le sens eschatologique et sotériologique de la présence impériale sur l'image est nettement suggéré. Dans le panneau de la galerie nord, antérieur aux deux derniers que nous venons de voir, Alexandre, frère de Léon VI, est représenté seul, avec des croix dans des cercles autour de lui (fig. 7). La croix est le symbole par excellence de la foi et particulièrement représentatif de l'imagerie déjà à l'époque iconoclaste. Ces croix sont aussi des images, reliées sémantiquement entre elles. Ainsi une prière est disposée dans ces croix et elle se prolonge de l'une à l'autre. La formule dit: "Seigneur, vient en aide à ton serviteur, orthodoxe, fidèle despote $\aleph^{48}$. La traditionnelle croix est reprise dans la nouvelle imagerie, mais elle est modifiée par la fonctionnalité qui lui est attribuée de signifier autre chose qu'auparavant. 
Fig.7

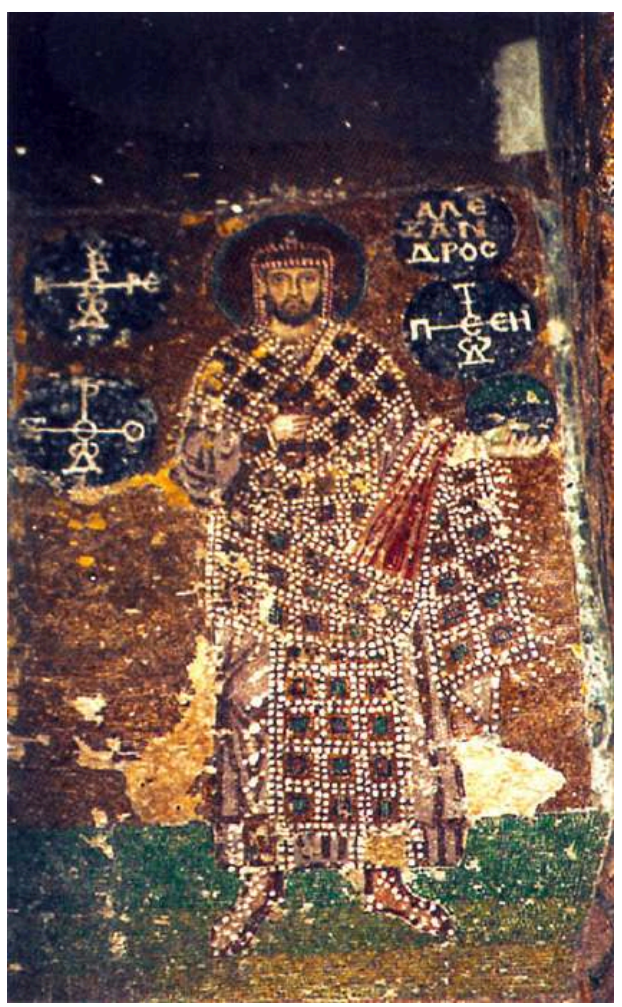

Alexandre. Tribune nord, Sainte-Sophie.

Il faut aussi insister sur la finalité des images. Si parfois, la représentation du souverain pouvait servir de repère temporel pour l'époque de construction ou de décoration de l'église, tel n'est pas le cas à Sainte-Sophie. Au contraire, les images (des basileis) n'étaient pas nécessairement destinées à être vues et regardées par les fidèles. A la différence des idées des théologiens iconodoules et de celles que Photius veut inculquer sur les images et leur utilité, les représentations des empereurs étaient, pour la plupart, cachées aux yeux des fidèles. Par conséquent, leur interprétation traditionnelle comme propagande est assez discutable. Elles semblent exclusivement destinées aux empereurs eux-mêmes, s'adressant plutôt à la divinité qui les accompagne. Le discours de Photius a pu influencer la réception des images. En célébrant l'inauguration de la mosaïque absidale de la Théotokos, il nous dit que les mosaïques sont l'œuvre d'empereurs pieux, mais, comme cela a déjà été suggéré, elles ont dû être pensées avant tout par les ecclésiastiques, et surtout par le patriarche. Seulement, l'empereur préside aux œuvres divines, comme lors des Conciles, et il est pris comme source, comme initiateur des dons.

Ainsi, plus qu'une décoration, les mosaïques peuvent être considérées comme des dons offerts au Christ et/ou à la Vierge pour manifester la fidélité des sujets. Cette conception rejoint toute la théorie des images qui sont dites, par les iconodoules, capables de créer des correspondances, d'" ouvrir des fenêtres ", entre la terre et le ciel. Dans la demeure divine sur terre, l'image envoie son message, sa prière de soutien et de salut au Christ et rend présent Dieu devant son élu. Un échange relationnel, un message communicationnel entre Dieu et son élu a lieu dans l'espace sacré des images. Une fois de plus, l'image témoigne de la vraie foi du souverain, de sa fidélité à 
l'orthodoxie, de son pouvoir dans le Christ et en son nom, du souci d'obtenir le salut promis.

Alexandre, aussi bien que le basileus anonyme (le basileus modèle par excellence), et les autres empereurs des mosaïques sont représentés, non pas tels qu'ils pouvaient se présenter à l'église, mais tels qu'ils apparaissaient sur l'itinéraire vers l'église et sur le retour au Palais, dans leurs costumes d'apparat selon le jour de la fête, comme le décrivait Constantin VII lui-même dans son Livre des cérémonies. D'ailleurs, la couronne reste en dehors de l'église, à l'exception de la cérémonie de couronnement, et la seule manière de la rendre présente est l'image. Adopter une attitude de prière et de respect, tenir la croix, offrir des dons, ces gestes avaient une efficacité particulière. Ils faisaient parvenir le don, en dehors de toute temporalité, et l'inscrivaient dans l'histoire universelle de la Rédemption par les actes. Sur l'image, un raccourci du Jugement dernier - la Déisis, au sens propre du mot, présente le Christ qui est à la fois trônant en Majesté et assis en Juge tenant le livre fermé ou ouvert, le Christ offert pour la Rédemption de l'homme et offrant lui-même la possibilité à l'homme (et à celui qui est à la tête du peuple chrétien, son roi) de se racheter par ses actions. L'image donnait aussi la possibilité aux sujets en général (et aux ecclésiastiques en particulier) de prier pour le salut de la personne représentée, pour le bienfaiteur de l'Eglise.

L'image qui mêle le temps de l'historia et de la memoria, et fait se côtoyer le surnaturel et l'humain place ainsi les personnages dans une perspective eschatologique. Le moment du Jugement dernier est implicitement présent et les souverains savent qu'ils seront jugés pour leurs actes, car si le pouvoir peut leur être accordé par grâce, le conserver est une question de mérite. Nul geste ne saurait échapper à la connaissance du Christ. Le livre est la Loi et il dicte le comportement à avoir pour mériter la vie éternelle. Le temps symbolique est ainsi celui du Jugement dernier, le jour où il faudra rendre des comptes $^{49}$. Le lieu symbolique de l'échange inscrit, quant à lui, l'image à la fois dans une temporalité historique et traditionnelle, mais la soustrait aussi au monde humain pour l'ouvrir à l'éternité promise. L'image ne s'intéresse à la temporalité, voire même au temporel, que dans la mesure où l'action humaine, marquée par le temps chronologique, liturgique, historique, s'inscrit dans l'histoire sainte, dans le temps de la Rédemption. L'image reste profondément imprégnée par les événements et les personnalités terrestres ; la tradition est élevée en culte et le passé est transformé, en même temps, selon les normes du présent; la temporalité est évacuée par le souci de l'éternel et de l'intemporel du modèle, orientée vers un devenir, celui qu'espère et craint tout fidèle.

De bafouée, l'image a gagné en légitimité pour devenir un don à Dieu (au Christ). Désormais, la foi peut s'exprimer à travers elle. Grâce à une manipulation de la tradition et à l'usage intentionnel du temps lors des cérémonies ou dans l'iconographie (faisant coïncider les temporalités humaine et divine), l'image s'est imposée (et a été imposée) pour célébrer le don de Dieu et le don à Dieu. 


\section{NOTES}

1. Ici, le terme d'image est à prendre au sens d'images saintes ou d'icônes. Pour approfondir l'interrogation sur la différence entre l'image et l'icône, v. A. Vasiliu, « Voir, nommer, regarder une image à la fin de l'Antiquité ", Miltos Garidês (1926-1996) afierôma, Panepistêmio Iôanninôn, Tomeas arhaiologias, Iôannina, 2003, p. 813-845, p. 817.

2. Cf. fig. 1 et 2 . Il faut noter que l'authenticité de la mosaïque est assez discutable. D. Talbot Rice discute la date de sa production et choisit de l'attribuer au XII ${ }^{\mathrm{e}} \mathrm{s}$. (Constantinople. Byzance-Istanbul, Paris, Albin Michel, 1965, p. 96). Les hésitations vont du IX ${ }^{\mathrm{e}}$ au XIV ${ }^{\mathrm{e}}$ siècles. Le visage de la Vierge semble refait. Cependant, le type de la Vierge à l'Enfant correspond bien au type avant l'iconoclasme. Au XII ${ }^{\mathrm{e}}$ siècle, il y a une préférence pour représenter la Vierge debout, mais un grand nombre de représentations géorgiennes, par exemple, suivent le même schéma. Cf. A. Eastmond, Royal imagery in medieval Georgia, The Pennsylvania State University Press University Park, Pennsylvania, 1998, pl. VI. Sur la nouveauté du programme décoratif, v. aussi R. Cormack, "Patronage and New Programs of Byzantine Iconography », The Byzantine Eye. Studies in Art and Patronage, London, Variorum Reprints, 1989, ch. X, p. 619.

3. Cf. les travaux de R. Scott de l'Université de Melbourne et ses conférences à l'Ecoles des hautes études en sciences sociales, en 2003.

4. Cf. fig. 2 .

5. Ou, selon une autre traduction, "Les icônes que les hérétiques ont jetées à terre, de pieux empereurs les ont relevées ». La première variante est selon S. Yerasimos, Constantinople. De Byzance à Istanbul, Paris, Editions Place des Victoires, 2000, la seconde - selon R. Cormack, Icônes et société à Byzance, Paris, Editeur Gérard Monfort, 1993 (pour la trad. fr.).

6. Cf. fig. 1 vs fig. 3 et 4 : on peut y comparer la conformité de la Théotokos avec les autres modèles pré-iconoclastes.

7. Le mouchoir, signe de deuil, est un symbole qui prend une nouvelle importance dans la pensée théologique en images. Il annonce en même temps la joie de l'Incarnation et les larmes de douleur du Sacrifice futur. Cet élément trouve une nouvelle promotion dans l'art monumental. Rares étaient les images à le présenter comme c'était le cas de l'image funéraire de Turtura des Catacombes de Commodille à Rome. Dans les représentations monumentales de la Vierge de l'époque avant l'iconoclasme, cet élément manque en général. Le type ancien de la Mère à l'Enfant est repensé et réactualisé dans un nouveau contexte. La composition se simplifie et trouve une place exceptionnelle pour Constantinople. Elle semble se conformer au passé et sous sa garantie actualise son iconographie.

8. Cf. R. Cormack (Icônes et société à Byzance) est sensible à ce déplacement du sens. Il suppose aussi que, par le rôle qu'ont joué les deux impératrices dans l'institution du culte des icônes, l'image de la femme est valorisée et la dédicace de l'église est faite à la Sagesse divine. Le premier édifice est dû à Constance II (commencé vers 350, et inauguré vers 360, dédié initialement au Christ, appelé la Grande Eglise), puis l'église est restaurée par Théodose II (415) et incendiée après la révolte de Nika en 532, inaugurée cinq ans plus tard, à Noël.

9. J. Durand signale cette pratique qui touchait l'ensemble de l'image, et non seulement les yeux, comme ce sera le cas plus tard : « Mais la piété populaire se mit bientôt à gratter des icônes pour en recueillir une poudre qui passait pour guérir tous les maux, et parfois des prêtres n'hésitaient pas à en mêler au sang du Christ dans le calice ", L'art byzantin, Paris, Terrail, 1999, p. 71.

10. Voici un passage de son discours «qu'ils [ceux qui blâment les iconodoules] sachent qu'au commencement Dieu fit l'homme à son image... mais parce que devenu véritablement, essentiellement homme, il a passé sa vie sur terre, s'est mêlé aux hommes, a fait ses miracles, a souffert, a été crucifié, ressuscité et a monté au ciel. Toutes ces choses sont arrivées réellement, 
les hommes les ont vues, on les a reproduites en dessins pour nous en souvenir et pour l'enseignement de ceux qui n'y ont pas assisté en sorte que sans les avoir vues, mais en écoutant et en croyant, nous retrouvions la joie du Seigneur. Comme tout le monde ne savait pas lire ou n'en avait pas le loisir, les Pères ont vu dans ces icônes comme un bref rappel de ces actions sublimes. Souvent, alors même que l'on ne pense pas à la passion du Seigneur, en voyant l'icône de la Crucifixion du Christ, elle nous revient mémoire et, tombant à genoux, nous adorons celui qui est reproduit et non la matière ; pas plus que nous n'adorons la matière des Evangiles ni la matière de la croix, mais l'image gravée dessus... La vénération pour elle rejaillit sur celui qui par elle s'est incarné. Pareillement les exploits de ces hommes saints nous poussent au courage, au zèle à imiter leur vertu et à chanter la gloire de Dieu. Comme nous le disons, la vénération pour la grandeur des serviteurs prouve nos bonnes pensées envers le commun Maître et la vénération de l'icône rejaillit sur le modèle. Il y a une tradition non écrite, comme d'adorer tourné vers l'Orient et beaucoup d'autres choses semblables ; c'est elle qui nous fait adorer la croix... Les apôtres ont laissé bien des traditions non écrites... »: J. Damascène, La foi orthodoxe, suivie de Défense des Icônes, préface de Jean Kovalevsky, traduction, introduction et notes docteur E. Ponsoye, publication de l'Institut orthodoxe français de théologie de Paris, Saint-Denys, diffusion Editions Cahiers St-Irénée, Paris, 1966, p. 184 et s. Toute une pensée philosophique antique est ainsi christianisée et mise au profit de la représentation. L'action du théologien commence déjà sous Léon III et se poursuit sous Constantin Copronyme.

11. Un fragment du texte du décret donne la mesure de leur usage: «... l'image de notre Seigneur, Dieu et Sauveur Jésus-Christ, celle de notre Dame immaculée, la sainte Mère de Dieu, celle des anges, dignes de notre respect, celle de tous les saints et justes. En effet, plus on les voit, grâce à leur représentation par l'image, plus en contemplant leurs images on est amené à se rappeler et à aimer les modèles originaux et à leur donner salutations et respectueuse vénération; non pas l'adoration véritable propre à notre foi, qui convient à la nature divine seule, mais comme on le fait pour la représentation de la glorieuse et vivifiante croix, pour les saints évangiles et tous les autres objets sacrés ; ... l'honneur rendu à l'image s'en va au modèle original »: G. Alberigo, Les conciles cecuméniques, Les décrets, tome II-1, Paris, Le Cerf, 1994. Un second iconoclasme est déclenché en 815 jusqu'au 11 mars 843.

12. Cf. pour ce rapprochement avec le labarum, A. Grabar, L'iconoclasme byzantin, Paris, Flammarion, 1984 ; cf. aussi le Christ est son image sur le mandylion donnée au roi de la ville d'Edesse Abgar (histoire que J. Damascène rapporte dans sa défense des images); ou encore l'icône de la Théotokos qui a sauvé du siège la ville de Constantinople, devenant sa protectrice. Suivant le modèle du labarum victorieux sur l'ennemi s'est constituée une tradition de l'image surnaturelle aidant à rapporter la victoire.

13. R. Cormack, Icônes et société à Byzance, op. cit., connue aujourd'hui grâce à deux manuscrits conservés l'un du IX ${ }^{\mathrm{e}}$, l'autre du XII ${ }^{\mathrm{e}}$ s., p. 134.

14. Sur l'image-corps, v. J.-Cl. Schmitt, Le corps des images. Essais sur la culture visuelle au Moyen Age, Paris, Gallimard, 2002 ; et l'article sur les «Images ", dans le Dictionnaire raisonné de l'Occident médiéval, (dir. J. Le Goff et J.-Cl. Schmitt), Paris, Fayard, 1999.

15. P. Brown, La société et le sacré dans l'Antiquité tardive, Paris, Ed du Seuil, pour la trad. fr. 1985 (original 1982), p. 199 et tout le chapitre «Une crise des Siècles sombres » y est consacré. Il est rejoint par M.-F. Auzépy, «Les enjeux de l'iconoclasme », Cristianità d'Occidente e cristianità d'Oriente, Spolète, avril 2003, Settimane di studio della fondazione centro italiano di studi sull'alto medioevo, Spolète, 2004, p. 127-169, qui partage le même avis sur l'iconoclasme.

16. Sur ces considérations v. plus en détail P. Brown, Société et sacré, op. cit., p. 203.

17. Cf. R. Cormack, Icônes et société à Byzance, op. cit., p. 165-174, Sermon $n^{\circ}$ XVII, prononcé le 29 mars 867, samedi de Pâques du haut de l'ambon au centre de la nef de Sainte-Sophie, en présence des coempereurs Michel III et Basile Ir à l'occasion du jour de l'inauguration de la mosaïque de la Mère de Dieu. 
18. Depuis N. Oikonomides (cité $\left.n^{\circ} 35\right)$, on pense que ce sont en effet les patriarches qui ont été à l'origine des mosaïques.

19. Cf. l'abside de S. Prudenziana à Rome du IV s., celles de S. S. Cosma e Damiano, à Rome, S. Vitale et $\mathrm{S}$. Apollinare in Classe à Ravenne, du VI ${ }^{\mathrm{e}}$ siècle, et d'autres. Dans l'art copte, la Vierge pouvait orner une abside, mais c'était plutôt celle des chapelles. Tel est aussi le cas à Kiti, sur l'Ile de Chypre. On imagine cependant plus facilement des similitudes entre Rome et Constantinople qu'entre centre et périphérie. Pour ces reproductions, v. A. Grabar, L'âge d'or de Justinien. De la mort de Théodose à l'Islam, Paris, Gallimard, 1966.

20. Cf. fig. 5-10.

21. Une représentation de Jean V Paléologue a été découverte, mais n'a pas encore été nettoyée. Déjà T. Velmans la mentionne dans La peinture murale byzantine à la fin du Moyen Age, (Paris, Ed. Klincksieck, 1947), de même que, plus tard, S. Yerasimos (dans Constantinople, op. cit., p. 101).

22. Cf. A. Grabar, L'iconoclasme, op. cit., p. 30-34, dont un ivoire où l'empereur est à cheval, le Christ bénit droit devant lui (cf. annexe 4 de l'ouvrage), et sur chacun des bras d'une croix des effigies enfermées dans un clipeus.

23. Cf. K. Weitzmann, "Les icônes de Constantinople ", dans Les icônes, Paris, Ed. de la Martinière, trad. fr. 1982 et 1992 (éd. orig. 1981), p. 14. Cf. aussi fig. 12 pour une reproduction du motif de la Vierge et des archanges avant l'iconoclasme.

24. C'est lui qui a mené les travaux lors de la découverte des mosaïques, voir ses rapports de recherches (en 4 vol. ) : Th. Whittemore, The Mosaics of St. Sophia at Istanbul. Preliminary report on the first year's work, 1931-2. The Mosaics of the Narthex, Paris, Oxford University Press of the Byzantine Institute, 1933 ; The Mosaics of St. Sophia at Istanbul. Second preliminary report work done in 1933 and 1934. The mosaics of the Southern Vestibule, Paris, Oxford University Press of the Byzantine Institute, 1936; The Mosaics of Haghia Sophia at Istanbul. Third preliminary report work done in 1935-1938. The imperial Portraits of the South Gallery, Boston, Oxford University Press of the Byzantine Institute, 1942. Cf. aussi les avis de C. Mango Materials for the Study of The Mosaics of St. Sophia at Istanbul, District of Columbia, The Dumbarton Oaks Research Library and Collection Trustees for Harvard University Washington, 1962 et T. Velmans (coll.), Le Rayonnement de Byzance, Paris, Zodiaque Desclée de Brouwer, 1999. L'analyse de l'image comme une prière et la promesse de salut qui en découle, pouvant donner courage au pénitent est faite par R. Cormack, "Patronage and New Programs of Byzantine Iconography», op. cit, ch. X, p. 622. G. Dagron suggère, à son tour, une interprétation de l'image comme étant celle de l'empereur pénitent après iconoclasme. Pourtant, dans l'histoire de la réception de l'image, la proskynèse et l'intercession sont reprises à une époque plus tardive (mil. $\mathrm{XI}^{\mathrm{e}} \mathrm{s}$.), notamment dans l'abside de Karanlik kilise : non seulement la position de prière aux pieds du Christ est reprise, mais aussi le texte du Livre du Christ (Jean VIII, 12 : "Je suis la lumière... »). Cf. pour une reproduction C. Jolivet-Lévy, Les églises byzantines de Cappadoce. Le programme iconographique de l'abside et de ses abords, Paris, Ed. du CNRS, 1991, p. 133, pl. 82. A une époque antérieure aussi (en 940), sur un autre support, cette posture était préférée pour la matérialisation de la prière et de l'hommage. Cf. aussi la Bible de Léon Sakellarios, Vat. reg. gr. I, BAV, 41x27 cm, 940, Constantinople, fol. 3r.

25. Il s'agit du sommet d'un sceptre, appartenant au Musée de Berlin (Staatliche Museen zu Berlin-Preußischer Kulturbesitz Museum für Spätantike und Byzantinische Kunst), inv. n. 2006. V. C. Jolivet-Lévy, «L'image du pouvoir dans l'art byzantin à l'époque de la dynastie macédonienne (867-1056) ", Byzantion, Bruxelles, t. LVII, 1987, p. 441-470, p. 446 et s., qui note que l'identité de Léon ne fait pas unanimité, mais qu'a priori, il s'agirait de Léon VI.

26. Cf. T. Velmans, Le Rayonnement, op. cit., p. 100 et s. Pourtant, Léon VI a écrit aussi bien des Homélies sur le Jugement dernier que sur l'Annonciation, mais il reste difficile de considérer les images comme des illustrations des écrits, surtout en présence d'un empereur anonyme.

27. L'archange conservé de l'abside montre une forte similitude avec celui du panneau du narthex. 
28. Un exemple intéressant de la réception plus tardive et de l'implication des anges peut être trouvé dans une église en Albanie actuelle, où le Christ Emmanuel, tenant le livre fermé, est entouré de deux anges. Tous les trois sont représentés dans des médaillons, mais les anges reprennent le geste, qui est ici celui de la Vierge, et lèvent les mains en signe de prière vers le Christ. (cf. Heideet Helmut Buschhausen, Die Marienkirche von Apollonia in Albanien. Byzantiner, Normannen und Serbien im Kampf um die Via Egnatia, Wien, Verlag des Österreichischen Akademie der Wissenschaften, 1976, tableau en couleur II, fig. 2). Les anciens gardes ont emprunté un comportement plus libre.

29. Une citation de l'Evangile de Jean y est inscrite : «Moi, je suis la lumière du monde. Qui me suit ne marchera pas dans les ténèbres, mais aura la lumière de la vie ». (Jn, $8: 12$ ). Devant la porte d'entrée vers le naos, sur une plaque en métal, en dessous de cette mosaïque (fig. 5), il y avait une autre invocation inscrite sur un livre ouvert, posé sur un trône, avec la colombe descendant sur lui : «... Moi, je suis la porte. Si quelqu'un entre par moi, il sera sauvé... » (Jn, 10 : 7-9). Pour une reproduction, voir aussi C. Mango, Hagia Sophia. A Vision for empires, Istanbul, Ertuğ et Kocabiyik, 1997 (en anglais avec de superbes photographies).

30. D’ailleurs une Déisis sous sa forme désormais traditionnelle avec saint Jean est représentée dans la galerie sud, datant d'avant 1300. Pour un détail de la Vierge et des anges, v. fig. 11 et 12 .

31. Pour une reproduction, v. G. Galassi, Roma o Bisanzio. I musaici di Ravenna e le origini dell'arte italiana, Roma, La libreria dello stato, 1953 (en 2 vol.), planche XXXI. A comparer avec la planche précédente mettant en image la Vierge, entourée de la même manière que le Christ trônant par des anges à l'église Saint-Apollinaire-le-Neuf.

32. L'interprétation du mot Déisis dépend de son acception concrète qui signifie " prière » (au sens propre du mot) et peut être comprise comme toute prière d'intercession auprès du Christ pour le pardon des péchés (comme l'entend A. Cutler, «Under the Sign of the Deēsis. On the Question of Representativeness in Medieval Art », Byzantium, Italy and the North. Papers on Cultural relations, Londres, The Pindar Press, 2000, ch. III, qui remet à plat la notion de Déisis dans l'iconographie en expliquant que différents contextes, usages et images ont la même désignation). Mais le terme a aussi une signification précise dans l'historie de l'art où la composition iconographique du Christ entouré de la Vierge (à gauche) et, généralement, de saint Jean (à droite), tous deux en prière, est appelée Déisis. Parfois, saint Jean Baptiste peut être remplacé soit par saint Jean le Théologien (et I. Spatharakis signale cette occurrence), soit par le patron de l'église (comme, par exemple, dans la basilique de Saint-Marc à Venise).

33. C. Jolivet-Lévy signale la Déisis sur une ampoule datant d'avant la période iconoclaste (cf. Les églises byzantines de Cappadoce, op. cit., p. 340, n. 28 et s.; pour la reproduction, cf. A. Grabar, Ampoules de Terre sainte (Monza-Bobbio), Paris, Librairie C. Klincksieck, 1958, p. 43 et., p. 60, pl. LIII). La composition peut être rapprochée aussi de l'Ascension avec la Vierge, saint Jean et Zacharie, son père, remplaçant les apôtres. Les personnages ne sont pas sur le même registre et une autre inscription explique le sens de l'image, tirée de Jean 1, 29 : «Voici l'agneau de Dieu... ». Même si A. Grabar la considère comme un hapax, il faut noter que C. Cecchelli (« Note iconografiche su alcune ampolle Bobbiesi », Rivista di archeologia Cristiana, Rome, $n^{\circ} 4$, 1927, p. 115-139) la compare, à juste titre, à un relief de la porte de l'église de Sainte-Sabine où la Vierge n'est plus en orante, mais tournée de trois-quarts et où la mandorle du Christ n'est pas porté par des anges mais par le tétramorphe. C'est plutôt ces éléments qui seront retenus pour figurer le Jugement dernier avec le Christ, dans sa mandorle, entouré de la Vierge et de saint Jean, au milieu des apôtres. Tania Velmans a pu attirer notre attention sur la «Déisis» de l'église de la Transfiguration du monastère de Sainte-Catherine au Mont Sinaï, datant du VI ${ }^{\mathrm{e}}$ s, mais là non plus, comme dans le cas de l'ampoule, il ne s'agit pas encore de la formule iconographique traditionnelle, consacrée seulement des années après la victoire des icônes. D'un autre côté, une Déisis égyptienne avec la Vierge, accompagnée non pas de saint Jean, mais d'un ange, serait mentionnée dans des textes des $\mathrm{V}^{\mathrm{e}}-\mathrm{VI}^{\mathrm{e}}$ siècles. (Cf. Le Rayonnement..., p. 90, n 141). Par contre, des Déisis commencent à être 
représentées à partir du $\mathrm{X}^{\mathrm{e}}$ siècle, comme celle du triptyque Harbaville où la Vierge est à la droite du Christ (!) (mil. X ${ }^{\mathrm{e}}$ s., ivoire, Musée du Louvre). L'iconographie ne suit pas strictement celle du Jugement dernier, mis à part la disposition tripartie - la Vierge, le Christ, saint Jean) : dans les compositions traditionnelles de Déisis, le Christ n'est pas dans une mandorle, mais sur un trône somptueux. Une autre différence consiste dans le fait que, dans les scènes du Jugement dernier, le Christ ne porte pas généralement de livre, mais a les bras ouverts vers les deux intercesseurs, alors qu'il a un livre - fermé ou ouvert dans la Déisis. La Cappadoce conserve de magnifiques exemples de Déisis. Un cas proche de celui qui nous intéresse se trouve sur une voûte de Göreme 33 (Meryemana) où la Vierge et saint Jean, chacun dans un médaillon avec les mains visibles, entourent le Christ, figuré plus important, mais aussi enfermé dans un clipeus. Cet exemple peut être significatif de la réception du modèle de Sainte-Sophie. Entre 976 et 1025, une autre décoration absidale est significative de la réception du modèle de la Déisis du narthex, sans une conformité parfaite avec celle-ci, et se trouve à Direkli Kilise, où le Christ central, trône en tenant le livre fermé, entouré d'anges et de la Vierge et de saint Jean, tandis que dans des médaillons sont représentés Pierre et Paul. Cette composition est plus tardive que celle de Sainte-Sophie et développe déjà ces premiers motifs. Plus tard, apparaissent même des personnages priant à ses pieds, comme dans l'abside de Karanlik Kilise. Ces compositions rappellent fortement la composition que nous analysons ici et expliquent son sens. C'est un témoignage précieux sur la réception et l'invention de modèles dans la durée. La fresque de Direkli Kilise nous donne aussi une limite chronologiquede la mosaïque dite de Léon, à savoir avant 976. A ce moment, le modèle était déjà imité et développé.

34. L'iconographie se cherche et s'invente dans un souci permanent d'afficher une fidélité à la tradition. $\mathrm{Au} \mathrm{XI}$ siècle, nous pouvons trouver une résonance de cette iconographie de SainteSophie qui a pu être reprise à Sainte-Sophie de Kiev avec une Déisis de la Vierge et déjà de saint Jean, entourant le Christ, présentés dans des médaillons, mais cette fois au-dessous de la conque de l'abside avec une Vierge orante sur fond d'or, affichant le même désir de tradition respectée et d'innovation cachée. Ce fait pourrait être une indication de la compréhension de la scène de la lunette du narthex que nous analysons, de sa réception et de sa modification un siècle plus tard. 35. N. Oikonomides, «The significance of some imperial monumental portraits of the $\mathrm{X}$ and XI centuries ", Zographe, Belgrade, 25, 1996, p. 23-26. Cependant, cela ne signifie pas non plus qu'un autre empereur n'aurait pas pu offrir la mosaïque à son prédécesseur, à travers un visage typologisé. Constantin VII, qui avait donné à la Grande église son Synaxaire, qui avait écrit des textes visant à créer un modèle aussi bien de bon gouvernement avec De Administrando Imperii, destiné à son fils, qu'une institution de rituel cérémoniel unique pour la cour avec De Ceremoniis, aurait aussi pu vouloir donner une image-modèle de la piété. Aussi, Constantin VII a pu vouloir rendre hommage aux prédécesseurs et donner un exemple aux successeurs. Il aurait pu aussi être à l'origine de la conception de la mosaïque.

36. Contrairement à l'argument de rivalité que semble avancer $\mathrm{G}$. Francastel, Le droit au trône. Un problème de prééminence dans l'art chrétien d'Occident du IV au XII siècle, Paris, Editions Klincksieck, 1973.

37. Dans les Homélies de Grégoire de Naziance, sur la miniature du ms. 510, Basile I $^{\text {er }}$ (867-886) est couronné par l'archange Gabriel et reçoit le labarum des mains de saint Elie, alors que le Christ trônant est peint sur un autre folio et n'est même pas en face de lui. Léon VI est, quant à lui, couronné par la Vierge sur un fragment de son sceptre, et ce n'est pas lui qui est central, mais elle. Constantin VII lui est couronné par le Christ sur une plaque d'ivoire, conservée à Moscou.

38. Cf. le chapitre sur Constantin le Grand de G. Dagron, Empereur et prêtre. Etude sur le " césaropapisme » byzantin, Paris, Gallimard, 1996.

39. Même s'il s'agit du lieu par lequel l'empereur entre pour assister à la liturgie, cette attitude n'est pas une illustration de la pratique rituelle. Le De Ceremoniis n'atteste pas toujours de génuflexion de l'empereur à cet endroit de l'église. (Dans le premier chapitre sur «ce qu'il faut 
observer lors de la procession à la Grande Eglise ", Constantin VII mentionne une triple proskynèse au seuil du naos, à un endroit qui devait se trouver en dessous de la mosaïque, alors que, par exemple, dans le chapitre 32 sur «ce qu'il faut observer à la fête et au cortège de la Sainte Naissance, selon la chair, du Christ ", il n'est plus question de proskynèse lors du passage dans le naos, mais uniquement d'une salutation entre le souverain et le patriarche avant l'arrivée aux portes impériales.) Par ailleurs, l'image n'est pas une reproduction mimétique de la réalité, car l'empereur, à cette époque, n'entrait jamais à l'église couronné. Il abandonnait sa couronne dans le vestibule pour la recevoir à la fin de la cérémonie, toujours en dehors de l'église. L'image est le seul lieu autorisé du port de la couronne à l'intérieur de l'église.

40. Cf. Th. Whittemore, The Mosaics of St. Sophia at Istanbul. Second preliminary report work done in 1933 and 1934. The mosaics of the Southern Vestibule, op. cit. Des avis contraires existent sur le motif du don des Mages et sa signification dans l'iconographie. Pour A. Grabar, il s'agit d'un emprunt de l'iconographie royale païenne, où le Christ reçoit l'hommage comme un souverain. La Vierge, le plus souvent sur son trône, reçoit les dons destinés à son fils. Une influence de l'art impérial a pu transformer le don des Mages en un hommage royal. Pour Th. Mathiews (cf. The Clash of Gods. A Reinterpretation of Early Christian Art, Princeton, New Jersey, Princeton University Press, 1993), il pourrait s'agir aussi d'une référence, païenne, mais aux magiciens et à la magie. En effet, les Mages ne rendraient pas un hommage, selon les normes de l'art impérial, mais seraient plutôt les figures des prêtres orientaux exerçant des fonctions magiques. Cette opposition peut être dépassée par l'analyse du geste comme ambivalent à la fois célébrant la royauté christique et annonçant le sacrifice à venir.

41. Sur ce glissement de sens, v. les travaux de R. Scott de l'Université de Melbourne.

42. A supposer qu'elle soit le produit de la pensée impériale, comme le suggérait Photius dans son sermon ; mais même si elle était l'œuvre d'un patriarche, ou d'autres sujets, l'intentionnalité de modèle est claire.

43. Déjà dans un contexte funéraire, un personnage laïc pouvait se trouver en présence de la Vierge, comme c'est le cas de Turtura des Catacombes de Commodille ou de la mosaïque de SaintVital avec un Christ trônant sur un globe et se voyant offrir le modèle de l'église et l'hommage du saint patron. Seulement, dans aucun de ces cas le contact n'est direct, mais il passe par une escorte d'anges et/ou de saints.

44. Cf. fig. 13 et 14 .

45. Jean Comnène et Irène flanquant la Vierge à l'Enfant, avec leur fils, Alexis, sur un panneau séparé, XII ${ }^{\mathrm{e}}$ siècle.

46. Contrairement à l'avis prédominant, nous pensons que le panneau de Zoé est aussi refait et qu'il existait un autre personnage en dessous. De cela témoigne l'inscription de Zoé qui a la même graphie que celle de la réécriture à laquelle a été soumise l'inscription de Constantin IX. Cela fait remonter sa composition à une époque antérieure au règne de Zoé et conteste l'originalité de la composition comme lui appartenant. V. notre article «L'image manipulée : considérations sur une mosaïque de Sainte-Sophie ", article à paraître dans le numéro « L'image abîmée », Images Revues, $\mathrm{n}^{\circ} 2,2005$ (publication en ligne).

47. Cf. De Ceremoniis témoigne d'une pratique d'échange de dons à la fin de la cérémonie religieuse, qui a lieu près de la relique de la margelle du puits où le Christ avait rencontré la Samaritaine, dans la cour de l'église. Les sacs que les souverains portent sont les apokombia, donnés au patriarche qui leur remettait de nouveau la couronne, enlevée lors de leur entrée au vestibule.

48. Pour la transcription en grec, v. P. A. Underwood and E. J. W. Hawkins. «The Mosaics of Hagia Sophia at Istanbul. The Portrait of the Emperor Alexander. A Report on Work Done by the Byzantine Institute in 1959 and 1960 ", Dumbarton Oaks Papers, The Dumbarton Oaks Research Library and Collection Trustees for Harvard University Washington, District of Columbia,15, 1961, p. 187-217, p. 192. En haut à droite, c'est le nom d'Alexandre qui règne seul entre 912-933 et est associé au 
pouvoir avec son frère, Léon VI, encore du vivant de son père Basile $\mathrm{I}^{\mathrm{er}}$; depuis 911 , Byzance avait trois empereurs Léon, Alexandre et Constantin VII encore enfant. Le sens de lecture de la phrase est, de haut à gauche, kupié bo(è)thei (to so) dou(l)o, en bas à gauche, orthodoxo, puis Alexandre, à droite en haut, et, finalement, en bas à droite, pisto despotè.

49. Sur la différence iconographique entre Jugement dernier à la fin des temps et Seconde Parousie, v. M. Angheben, «Les Jugements derniers byzantins des $\mathrm{XI}^{\mathrm{e}}-\mathrm{XII}^{\mathrm{e}}$ siècles et l'iconographie du jugement immédiat », Cahiers archéologiques, Paris, 50, 2002, p. 105-134, p. 129.

\section{INDEX}

Mots-clés : post-iconoclaste, théophanie, iconodoulie, prototype, don, temporalité universelle

\section{AUTEUR}

\section{TANIA KAMBOUROVA}

Docteur de l'Ecole des Hautes Etudes en Sciences sociales, spécialité " Histoire et civilisations ». Sa thèse s'intitule " Le don dans l'image byzantine du souverain ». Il s'agit d'une thèse en cotutelle, sous la direction de Jean-Claude Schmitt (EHESS) et d'Elka Bakalova (Université de Sofia). Elle est actuellement en post-doctorat au GAHOM (EHESS) où elle poursuit ses recherches sur la notion de don dans la tradition byzantine. 